\title{
Competition between shocks and entropy floor: Unifying groups and clusters of galaxies
}

\author{
S. Dos Santos ${ }^{1}$ and O. Doré ${ }^{2}$ \\ 1 Department of Physics \& Astronomy, University of Leicester, 1 University Road, Leicester LE1 7RH, UK \\ e-mail: ssa@star.le.ac.uk \\ 2 Institut d'Astrophysique de Paris, 98bis boulevard Arago, 75014 Paris, France
}

Received 27 June 2001 / Accepted 6 December 2001

\begin{abstract}
Semi-analytic models of X-ray clusters and groups of galaxies, relying on the idea that there was a nongravitational energy injection in these systems, are able to reproduce many observed correlations, in particular the $L_{\mathrm{X}}-T$ relation and the "central entropy floor" in groups. Limiting models exist which describe the behaviour of clusters and groups separately, but no analytic modeling has yet been found to unify both mass ranges. It is the aim of this paper to provide such an analytic model. Our description relies on a now standard description of the shock thought to occur in these systems near the virial radius (Cavaliere et al. 1998), the isothermality and spherical symmetry of the intracluster medium, as well as the reinterpretation of observed quantities (like the X-ray luminosity, the gas mass $M_{\mathrm{ICM}}$ and the central SZ effect $\left.y_{0}\right)$ in terms of the specific entropy. This allows the derivation of analytic expressions for several observed correlations $\left(L_{\mathrm{X}}-T, M_{\mathrm{ICM}}-T, y_{0}-T, \ldots\right)$ and their normalisation encompassing both the group and the cluster regimes. The analytic predictions compare very well with observations, as well as with more elaborated semi-analytic schemes. This agreement allows a reinterpretation of the $L_{\mathrm{X}}-T$ relation (via the quantity $L_{\mathrm{X}} / T^{7 / 2}$ ) and the $y_{0}-T$ relation (via $y_{0} / T^{5 / 2}$ ) as indirect measures of the non-gravitational entropy content of groups and clusters of galaxies. We emphasize the need for shock heating, even in the group mass range: shocks can not be completely suppressed in groups (and thus groups can not be entirely isentropic) unless an unacceptably high entropy floor is needed in order to break the self-similarity in the $L_{\mathrm{X}}-T$ relation. Our model shows that the normalisation of the entropy after the shock (which is mass-dependent) is a key ingredient and that this quantity alone can explain the shape of the observed correlations between integrated X-ray and SZ quantities over and below $2 \mathrm{keV}$.
\end{abstract}

Key words. hydrodynamics - shock waves - galaxies: clusters: general - X-rays: galaxies: clusters - cosmology: large-scale structure of Universe

\section{Introduction}

For ten years, it has been known that X-ray observations of clusters of galaxies can not be reproduced in simple selfsimilar models, where the central gas density is proportional to dark matter density. Kaiser (1991) and Evrard \& Henry (1991) advocated a preheating of the gas before it fell into the cluster potential to recover the observed correlations. Later, X-ray observations of groups of galaxies have strengthened the case for a non-gravitational entropy injection in these systems, in particular by the observation of the so-called "entropy floor" (Ponman et al. 1999). These authors showed that the central entropy level in groups (outside the cooling-flow radius) is higher than the level the sole cosmological shocks can provide, while being well in accord with adiabatic numerical simulations (e.g.,

Send offprint requests to: S. Dos Santos, e-mail: dore@iap.fr including no dissipative physics) in clusters. This entropy injection could be due to supernovae explosions (the socalled feedback from star formation) or other sources as active galactic nuclei. Several semi-analytic schemes that elaborate on this idea have been proposed to reproduce the curvature of the $L_{\mathrm{X}}-T$ relation in the groups' mass range (where the effects of the preheating are thought to be the highest, see Cavaliere et al. 1997; Bower 1997; Valageas \& Silk 1999; Valageas \& Schaeffer 2000; Wu et al. 2000; Bower et al. 2000). In particular, Cavaliere et al. $(1997,1998,1999$, hereafter CMT) have introduced a simple model, where the bending is provided by the differential strength of a shock occurring at the virial radius in a preheated gas. Their description relies on the physical modeling of the shock interface, using Hugoniot relations, and reproduces naturally the central observed entropy floor. While this model highlights the important role of shocks and entropy floor in the formation of clusters 
and groups, no analytic description of the X-ray observed relations has yet been found to encompass both groups and clusters. The aim of this paper is to present such a model and compare it to observations.

However, despite the success of these semi-analytic schemes when confronted with observations, difficult problems remain, as for example the fact that a reheating by supernovae (hereafter SN) explosions requires an incredibly high efficiency of the transfer of energy from SN remnants to the intergalactic medium (Valageas \& Silk 1999; Bower et al. 2000). In particular, the cooling of the SN remnants will obviously decrease this transfer efficiency, and must be ignored in these models. While a combination of SN and quasars (hereafter QSOs) reheating is probable and would alleviate this problem, Bryan (2000) has proposed that differential galaxy formation between groups and clusters (which would lower the central entropy in groups and allow higher entropy gas to flow into the center) can explain the curvature of the $L_{\mathrm{X}}-T$ relation and the entropy floor. However, it is well known that without preheating, most of the available gas in the universe would have formed stars today, which is not observed (this the so-called "overcooling problem", see Blanchard et al. 1992; Balogh et al. 2001). This much-needed feedback in galaxy formation would certainly have an impact on the formation of clusters and groups. Valageas \& Silk (1999) have indeed shown that galaxy and cluster feedback differing requirements are likely to provide a tight constraint on the amount of preheating in the universe. But, even in the preheated scenarios, there is actually no consensus on the entropy injection epoch. While most of the studies have focussed on "external preheating" models (where the entropy injection occurs before the formation of groups and clusters e.g., before the gas is compressed by shocks) because low density gas requires much less energy than high density one to be raised to a given entropy level (and also because the star formation rate seems to peak rather early in the universe evolution), Loewenstein (2000), from a series of approximate static hot gas models and Brighenti \& Mathews (2001), from 1D numerical simulation with cooling, mass dropout and star formation feedback, have argued that most of the heating occurred during or after the assembly of the group or cluster gas. The efficiency problem of SN explosion is still present, but can be alleviated by an initial mass function flatter than the Salpeter one (Brighenti \& Mathews 2001). These are the so-called "internal preheating" models.

In this paper, we focus explicitly on an external preheating model. Internal preheating models require spatially-dependent and time-dependent star formation rates to provide the amount of injected entropy, while external preheating models only require the level of entropy before the gas falls in a cluster or a group. This simplification allows us to obtain a completely analytic model that describes both groups and clusters. The physical approach we follow consists of deriving scaling relations, e.g. $L_{\mathrm{X}}-T$, $y-T, y-L_{\mathrm{X}}$ by linking these observed quantities to the specific entropy profile in the system. For this purpose we first derive an analytic expression for the entropic profile normalisation at the virial radius of clusters and groups by discussing shocks, as in CMT. Using this relation we then derive analytically the relevant scaling relations and compare them to data. Following Tozzi \& Norman (2001), we highlight the key role of entropy and argue that it indeed constitutes the best observable in clusters, allowing to derive analytical expressions for standard X-ray and Sunyaev-Zeldovich (hereafter SZ) correlations. This leads us to reinterpret these relations in terms of global entropy content in a well physically motivated manner.

The plan of the paper is as follows: in Sect. 2, we present the shock model and derive an expression for the normalisation of the entropy profile at the virial radius. This expression, containing a free normalisation, is fitted to observations. In Sect. 3, we relate the X-ray luminosity to the entropy profile and derive an analytic expression for the shape of the $L_{\mathrm{X}}-T$ relation, which is subsequently compared to observations using only the fitted parameter of the last section.. The same method is followed in Sects. 4-6 to obtain analytic expressions for the $M_{\mathrm{ICM}}-T$, $f_{\text {gas }}-T, y_{0}-T$ and $y_{0}-L_{x}$ correlations (where $M_{\mathrm{ICM}}$ is the gas mass, $f_{\text {gas }}$ is the gas fraction and $y_{0}$ is the central Compton parameter). In each of these parts, we provide a detailed comparison with previous work. Section 7 discusses a reinterpretation of these correlations in terms of the total entropy content in groups and clusters, tests it using a semi-analytic model and discusses the main hypotheses we make. Moreover, a comparison of our results for the entropy floor with other theoretical models is made. Section 8 summarises the present work and concludes. We also compute the numerical values of the normalisations of the correlations found and summarise all these results in Appendix A, allowing them to be easily used in another context. Appendix B computes the infalling velocity as a function of the mean mass of the system and compares it to hydrodynamic simulations. Finally, Appendix C, introducing a simple model for the entropy profile of groups, computes the shape factors appearing in the different normalisations.

Unless otherwise stated, we use $H_{0}=$ $100 h_{2 / 3} \mathrm{~km} \mathrm{~s}^{-1} \mathrm{Mpc}^{-1}$ with $h_{2 / 3}=2 / 3$, which gives approximately $H_{0}=67 \mathrm{~km} \mathrm{~s}^{-1} \mathrm{Mpc}^{-1}$. We rescale all the data used in this paper to this value.

\section{Entropy and shocks in clusters and groups}

The study of the equilibrium state of X-ray clusters and groups of galaxies requires the physical description of the complex interplay between baryonic and non-baryonic dark matter. To tackle this problem analytically requires several simplifying hypotheses. The following assumptions are reasonable and will be thus assumed henceforth (see Sarazin 1988, for a review and a discussion of the main assumptions). 
First, the hot and diluted plasma of ions and electrons constituting the intracluster medium (hereafter ICM) is treated as a single species perfect gas satisfying

$P=\frac{\rho k T}{\mu m_{\mathrm{p}}}$,

where $P, \rho, T$ denote respectively the pressure, density and temperature of the baryonic gas, while $k$ and $\mu$ denote respectively the Boltzmann constant and the mean mass per proton. Second, neglecting the ICM mass with regards to the DM mass, and assuming stationarity (no gravitational potential variation on time scales smaller than the hydrodynamic time scale, e.g. no recent mergers), the ICM is assumed to be in hydrostatic equilibrium (hereafter HSE) in the dark matter gravitational potential $\phi$. We can thus write the continuity and the Euler equation as follows:

$\nabla(\rho v)=0$

$\nabla P=-\rho \nabla \phi$

where $v$ is the gas velocity and $\phi$ the gravitational potential. Equations (2), (3) and (1), together with the energy conservation equation and Poisson equation (the use of which can be avoided if an approximate analytic expression for $\phi$ is chosen, e.g., neglecting the gravitational contribution of the baryons) form a closed set of equations, whose resolution provides the radial profiles of all the quantities of interest in the gas.

To solve this set, boundary conditions are required. In the present paper, we are interested in the variations of these boundary conditions with the total mass of the system (or equivalently with its mean temperature). Indeed, the previous equations will describe the equilibrium of baryonic gas in the underlying potential, whatever the total mass of the system. We argue and show in the following that changes in the boundary conditions, when explicitly written as a function of the mean temperature, allow us to derive the change in global correlations, such as the $L_{\mathrm{X}}-T$ relation, on a scale going from clusters to groups of galaxies. For this purpose, we use the fact that the flow of gas on a cluster becomes supersonic in the vicinity of the virial radius $r_{\mathrm{v}}$ (Teyssier et al. 1997). We will then show that a standard modeling of the resulting shock (following CMT) leads to the appropriate boundary conditions. We will consider hereafter that the boundary conditions take place after the last major merger of the system, as appropriate in a hierarchical picture of structure formation.

The last hypothesis we will use is that within the virial radius, the ICM is isothermal. This hypothesis will not be used in the derivation of the boundary conditions (Sect. 2.1) but in the derivation of the global correlations in groups and clusters (Sects. 3 and 5). Both observations Markevitch et al. (1998) and simulations (Teyssier 2001) show that this approximation is good into a factor two and is thus sufficient for our purpose.

Finally, let us recall an hypothesis necessarily underlying every work published using the entropy of the ICM, but rarely stated: the local thermodynamic equilibrium hypothesis (hereafter LTE). By definition, in a state of global thermodynamic equilibrium, temperature and pressure (and thus density) are constant throughout the system, and the state functions of the system (e.g. entropy) have a determined dependency on the state variables chosen (and so a fixed value). In the LTE however, temperature and pressure can vary from point to point (as is obvious in clusters from X-ray observations for example) and, locally only, the system is in thermodynamical equilibrium. Thus, the state functions have the same dependency on state variables as in global equilibrium, but vary now from point to point as do the latter. Note that this hypothesis is likely to break down at smaller scales than the ones of interest to us, due to turbulence or magnetic effects. This hypothesis is crucial if we want to use the usual analytic expression of the specific entropy of the ICM, $s$, defined as

$s=S / c_{\mathrm{v}}=\ln \left(\frac{k T}{\rho^{\gamma-1}}\right)$

where $\gamma$ is the polytropic index of the gas. The thermodynamical state of the gas is fully known as soon as the entropy profile of the gas is known. Since we want to highlight the key role of entropy as "an observable" we choose to describe the physical state of clusters in terms of entropy, or more appropriately and equivalently in terms of the adiabat, defined as

$K=\left(\mu m_{\mathrm{p}}\right)^{\gamma-1} \mathrm{e}^{s}=\frac{k T}{n^{\gamma-1}}=(k T) n^{-2 / 3}$,

where $n=\rho /\left(\mu m_{\mathrm{p}}\right)$. In the following, $T$ will denote the temperature in $\mathrm{keV}$ (i.e., we will replace everywhere $k T$ by $T$ ). Note that from now on the polytropic $\gamma$ index will be fixed to its standard value of $5 / 3$. The definition of the adiabat differs from the widely used definition of Balogh et al. (1999) (by a constant factor $\left(\mu m_{\mathrm{p}}\right)^{\gamma-1}$ ) but allows a direct comparison to observations.

\subsection{A general shock model}

We first aim at deriving adiabat boundary conditions based on the Rankine-Hugoniot relations, used in the same form as CMT.

\subsubsection{The Rankine-Hugoniot relations}

Let the infalling gas velocity reach the sound speed at $r=$ $r_{\mathrm{v}}$ and consider subsequently that a shock forms at this radius. Let the temperature, the density and the velocity of the infalling gas before the shock be respectively $T_{1}$, $n_{1}$ and $v_{1}$, and the post-shock temperature, density and velocity be $T_{2}, n_{2}$ and $v_{2}$. If the shock efficiency is very high, i.e. the post-shock velocity $v_{2}$ is null in the restframe of the cluster center of mass, the Rankine-Hugoniot relations yield (see CMT):

$k T_{2}=\frac{\mu m_{\mathrm{p}} v_{1}^{2}}{3} F(\epsilon)$ 
with

$F(\epsilon)=\frac{(1+\sqrt{1+\epsilon})^{2}}{4}+\frac{7}{10} \epsilon-\frac{3}{20} \frac{\epsilon^{2}}{(1+\sqrt{1+\epsilon})^{2}}$

and

$\epsilon=\frac{15}{4} \frac{k T_{1}}{\mu m_{\mathrm{p}} v_{1}^{2}}$.

At the same time, the ratio between post-shock $\left(n_{2}\right)$ and pre-shock $\left(n_{1}\right)$ density is given by:

$\frac{n_{2}}{n_{1}}=2\left(1-\frac{T_{1}}{T_{2}}\right)+\left[4\left(1-\frac{T_{1}}{T_{2}}\right)^{2}+\frac{T_{1}}{T_{2}}\right]^{1 / 2}$.

Note that since the ICM within $r_{\mathrm{v}}$ is assumed to be isothermal, its temperature is equal to the temperature $T_{2}$ after the shock.

Using the formulas above, we will now derive a general expression for the adiabat jump at the shock, which will depend on the system mean temperature. This virial adiabat normalisation will provide us with the change in the boundary conditions necessary to describe groups and clusters in a unique analytic model.

\subsection{The adiabat after the shock}

To find a general expression for the post-shock adiabat, we first express the ratio $T_{1} / T_{2}$ as a function of $\epsilon$ :

$\frac{T_{1}}{T_{2}}=\frac{4}{5} \frac{\epsilon}{F(\epsilon)}$.

Introducing this expression in Eq. (9), one has:

$$
\begin{aligned}
n_{2}= & 2 n_{1}\left(\left(1-\frac{4}{5} \frac{\epsilon}{F(\epsilon)}\right)\right. \\
& \left.+\left[\left(1-\frac{4}{5} \frac{\epsilon}{F(\epsilon)}\right)^{2}+\frac{\epsilon}{5 F(\epsilon)}\right]^{1 / 2}\right) .
\end{aligned}
$$

Using then Eqs. (6), (8) and (11), the post-shock adiabat $K_{2}$ is easily expressed as a function of the pre-shock adiabat $K_{1}$ and $\epsilon$ in the following manner:

$$
\begin{aligned}
K_{2} & =\frac{k T_{2}}{n_{2}^{2 / 3}} \\
& =\frac{5}{2^{8 / 3}} K_{1} H(\epsilon),
\end{aligned}
$$

where

$$
\begin{aligned}
H(\epsilon)= & \frac{F(\epsilon)}{\epsilon}\left(\left(1-\frac{4}{5} \frac{\epsilon}{F(\epsilon)}\right)\right. \\
& \left.+\left[\left(1-\frac{4}{5} \frac{\epsilon}{F(\epsilon)}\right)^{2}+\frac{\epsilon}{5 F(\epsilon)}\right]^{1 / 2}\right)^{-2 / 3} .
\end{aligned}
$$

Even if this last expression looks complicated, it can be straightforwardly expanded in a Laurent series in the vicinity of $\epsilon=0$. We obtain:

$H(\epsilon)=\frac{17}{10} 2^{-2 / 3}\left(1+\frac{10}{17} \frac{1}{\epsilon}\right)+\mathcal{O}(\epsilon)$.
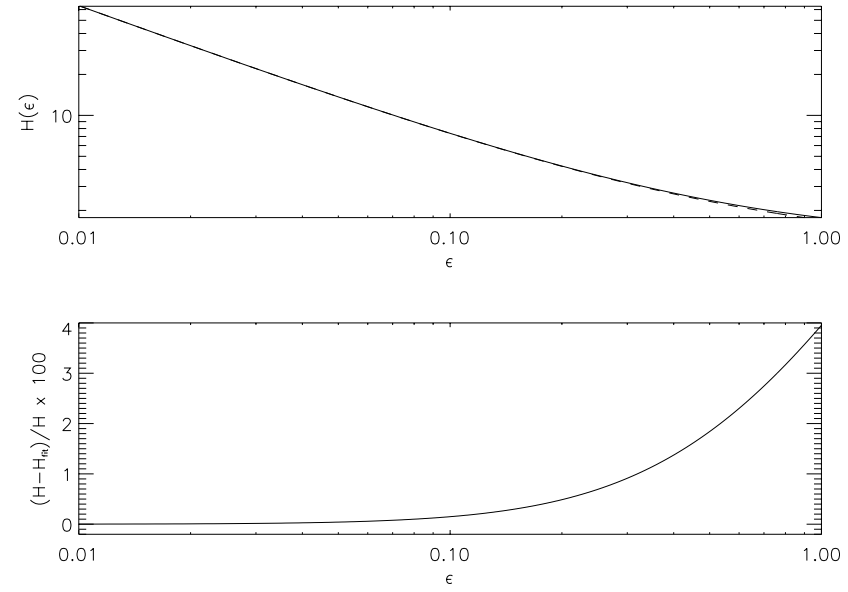

Fig. 1. Top: plot of the function $H(\epsilon)$ (Eq. (13), solid line), together with the two firsts order of its expansion (Eq. (14), dashed line). Both functions are hardly distinct. Bottom: Percentage difference between $H$ and its expansion. The agreement is better than $4 \%$ in the relevant $\epsilon$ range (and always better than $15 \%$ for all values of $\epsilon$ ).

Checking the validity of this expansion in the range $0 \leq \epsilon \leq 1$ we find an agreement better than $4 \%$ (Fig. 1) which is enough for our purpose (the error of the approximation reaches a constant $15 \%$ level at large $\epsilon$ ). Thus we will neglect higher order corrections.

As a consequence, for the all range from groups to rich clusters, one can write to a very good approximation:

$K_{2}=\frac{17}{16} 2^{-1 / 3} K_{1}\left(1+\frac{10}{17} \frac{1}{\epsilon}\right)$.

Note that this expression that fits so well the general adiabat expression is not really a surprise. Its physical significance is indeed straightforward. To show this, let us take two limiting behaviour, namely in the cluster and in the group mass range.

Since rich clusters of galaxies accrete mainly small clumps and diffuse gas, the cold inflow or strong shock limit is appropriate. Consequently it is usually argued that the entropy of rich clusters is dominated by shock created entropy. Indeed, for this approximation we have $T_{2} \gg T_{1}$ and $\epsilon \ll 1$, and so (from Eq. (15))

$K_{2} \simeq \frac{5}{8} \frac{2^{-1 / 3}}{\epsilon} K_{1}$.

On the other hand, for groups of galaxies, the weak shock or adiabatic infall approximation is more appropriate. Indeed since the infall speed tends to be lower, and since groups accrete preheated gas, the weak shock limit (whose limit is the true adiabatic infall) is appropriate $(\mathrm{CMT})^{1}$. Indeed, taking the limit $\epsilon \rightarrow 1$, Eq. (15) yields

$K_{2} \simeq \frac{27}{16} 2^{-1 / 3} K_{1}$

${ }^{1}$ Note that this hypothesis of adiabatic infall has been shown to reproduce the $L_{\mathrm{X}}-T$ relation in the group range by Balogh et al. (1999). 
This means that the entropy content of groups is dominated by the "adiabatically accreted" gas, hence the existence of a so-called entropy floor (the last expression of $K_{2}$ being independent of $\epsilon$ ).

The analytic expression in Eq. (15) thus provides a well physically motivated expression for the competition between shocks and entropy floor that rules the adiabat virial normalisation from groups to clusters.

\subsection{Linking groups and clusters}

\subsubsection{Defining a general analytic $K-T$ relation}

We now want to make use of the previously derived expression (15) to predict a general relation for the $K-T$ relation for groups and clusters.

First, making use of Eqs. (8) and (15) we rewrite

$K_{2}=\frac{17}{16} 2^{-1 / 3} K_{1}\left(1+\frac{8}{51} \frac{\mu m_{\mathrm{p}} v_{1}^{2}}{k T_{1}}\right)$.

Second, we need to express the infall velocity $v_{1}$ as a function of the mean temperature of the system. This is done in Appendix A, where we show that using the virial theorem, the $M-T$ relation, together with the assumption of adiabatic cold inflow, we can write $\left(\phi_{1}\right.$ being a characteristic virial radius gravitational potential, $T_{*}$ a characteristic temperature and $\eta$ a dimensionless constant, see Eqs. (B.5), (B.11) and (B.13)):

$v_{1}^{2}=-2 \eta \phi_{1}\left(\frac{T}{T_{*}}\right)$.

Note that from now on we will assume notations of Appendix A.

This leads naturally to:

$K_{2}(T)=\frac{17}{10} 2^{-2 / 3} K_{1}\left[1-\frac{16}{51} \eta \frac{\mu m_{\mathrm{p}} \phi_{1}}{k T_{1}}\left(\frac{T}{T_{*}}\right)\right]$

that we rewrite

$K_{2}(T)=K_{0}\left[1+\left(\frac{T}{T_{0}}\right)\right]$,

where we have defined ${ }^{2}$

$K_{0}=\frac{17}{16} 2^{-1 / 3} K_{1}$

and

$T_{0}=-\frac{51}{16 \eta} \frac{k T_{1}}{\mu m_{\mathrm{p}} \phi_{1}} T_{*}$.

The scaling relation (21) is a key result of our paper. It states how the entropy jumps due to the shock changes with the mean temperature of the system. Written this way, the last formula has two free parameters: a normalisation parameter $K_{0} \simeq K_{1}$, i.e. the adiabat before the shock, and the temperature $T_{0}$ that marks the transition from the adiabatic inflow to the strong shock regime.
Deriving the value of $K_{1}$ requires a whole semi-analytic scheme, which would require a model of the entropy evolution of the intergalactic medium (hereafter IGM) as the universe evolves (see e.g. Valageas \& Silk 1999). This is clearly beyond the scope of the present paper, and we will thus constrain $K_{1}$ by comparison with observations (which only means that the normalisation of our model is left free). However, $K_{0}$ and $T_{0}$ are not independent parameters and can be related by simple physical considerations as will be shown in the next section.

Once this is done, the scaling relation (21) is left with only one normalisation parameter $K_{0}$.

\subsubsection{Relating $K_{0}$ and $T_{0}$}

First, to derive an explicit relation between $K_{0}$ and $T_{0}$, we need to specify the value of both $\eta$ and $T_{1}$ in Eq. (23). Using the results of Miniati et al. (2000) for the infall velocity in hydrodynamic simulations (see Eq. (B.15) and Appendix for notations), a value for $\eta$ can be derived by equating Eq. (B.15) and Eq. (19):

$\eta=-\frac{v_{\mathrm{s} 0}^{2}}{2 \phi_{1}}\left(\frac{T_{*}}{T_{\mathrm{s}}}\right)$

which, when introduced in Eq. (23), gives:

$T_{0}=\frac{51}{8} \frac{k T_{1}}{\mu m_{\mathrm{p}} v_{\mathrm{s} 0}^{2}} T_{\mathrm{s}}$

where $T_{\mathrm{s}}$ and $v_{\mathrm{s} 0}$ are known (Appendix $\mathrm{A}$ ).

The relation between $K_{0}$ and $T_{0}$ will appear when expliciting $T_{1}$. Indeed we can write it as a function of the pre-shock adiabat $K_{1}$ :

$T_{1}=K_{1} n_{1}^{2 / 3}$.

The overdensity that eventually collapses and reaches a density $\rho_{1}$ at $r_{\mathrm{v}}$ has decoupled from the Hubble expansion at a turn-around redshift $z_{\text {ta }}$ where its density was $\rho_{\mathrm{ta}}$ and its radius was $r_{\mathrm{ta}}$. Following Balogh et al. (1999), we assume first that the IGM was preheated (non-gravitationally) before $z_{\text {ta }}$ and its adiabat raised to the value $K_{1}$. Second, we also assume that between $r_{\text {ta }}$ and the shock radius $r_{\mathrm{v}}$ itself, the gas has an isentropic behaviour. To fix the value of the overdensity (and thus the density) $\delta_{\text {ta }}=\left(\rho_{\text {ta }}-\bar{\rho}\right) / \bar{\rho}$ at the turn-around, we will consider the simplest collapse model, i.e. the spherical collapse model in a given cosmology.

By definition of the baryonic fraction $f_{\mathrm{B}}$ and the overdensity $\delta_{\text {ta }}$ we can write:

$\rho_{\mathrm{ta}}=\left(1+\delta_{\mathrm{ta}}\right) \rho_{\mathrm{c}} f_{\mathrm{B}}$

where $\rho_{\mathrm{c}}$ is the critical density. Inserting this last equation in Eq. (25), one obtains:

$T_{0}=\frac{51}{8} \frac{K_{1}\left(\left(1+\delta_{\mathrm{ta}}\right) \rho_{\mathrm{c}} f_{\mathrm{B}}\right)^{2 / 3}}{\left(\mu m_{\mathrm{p}}\right)^{5 / 3} v_{\mathrm{s} 0}^{2}} T_{\mathrm{s}}$,

which relates $K_{0}$ and $T_{0}$ via Eq. (22). 
Consequently, to fix the numerical value of $T_{0}$ we need to specify a value of $K_{1}$. We choose to assume the value of the observed entropy floor visible in groups of galaxies. A value of $K_{1}=120 \mathrm{keV} \mathrm{cm}^{2}$ is inferred from the data (Lloyd-Davies et al. 2000, see the next section).

Then we assume a flat $\Omega_{\mathrm{m}}=1$ universe with $H_{0}=50 \mathrm{~km} \mathrm{~s}^{-1} \mathrm{Mpc}^{-1}$ and the baryonic fraction as fixed by Big-Bang Nucleosynthesis standard, i.e. $f_{\mathrm{B}}=$ $0.015 h_{100}^{-2} / \Omega_{\mathrm{m}}$ (Olive et al. 2000). For a spherical collapse model $\delta_{\mathrm{ta}}=9 \pi^{2} / 16$. Note however that in the widely used hierarchical structure formation paradigm, this monolithic formation model is too simplistic. Indeed, since the majority of the infall of group of galaxies occurs through filaments, an overdensity of $\delta_{\mathrm{ta}} \sim 10$ should be more appropriate. Considering then the values of $v_{\mathrm{s} 0}$ and $T_{\mathrm{s}}$ specified in the Appendix, we find that $T_{0}=1.86 \mathrm{keV}$ for $\delta_{\mathrm{ta}}=9 \pi^{2} / 16$ and $T_{0}=2.62 \mathrm{keV}$ for $\delta_{\mathrm{ta}}=10$. This is quite insensitive to the adopted cosmology, since for an $\Omega_{\mathrm{m}}=0.3$ universe, one finds $T_{0}=1.71 \mathrm{keV}$ and $2.33 \mathrm{keV}$ respectively. It is thus obvious that we can take for $T_{0}$ an intermediate value between these two extremes.

In the following, we will thus consider that $T_{0}$ is fixed at $2 \mathrm{keV}$, and that the only free parameter of the model will be the normalisation factor $K_{0}$. Moreover, choosing a value of $T_{0}$ between 1.5 and $2.5 \mathrm{keV}$ does not alter the quality of the fits we present in the next sections.

Looking at Eq. (21), $T_{0}$ has a simple physical interpretation: it is the system mean temperature where the effect of an entropy floor begins to change the adiabat jump during the virial shock. For systems with $T<T_{0}$, the pre-shock entropy is high enough to reduce the shock and a quasi-adiabatic inflow takes places (but the shocks are not completely suppressed, see Sect. 7.4). For $T>T_{0}$, the shock adiabat jump brings the post-shock adiabat to values much higher that the entropy floor, the latter having less and less incidence as $T$ increases. Thus we find that this transition occurs around $2 \mathrm{keV}$.

\subsubsection{Comparing with observations}

X-ray observations of groups and clusters of galaxies have brought a great amount of information on the gas equilibrium in these systems. Unfortunately, due mostly to the high background of the instruments and the gas density decline with radius, there are not yet entropy observations available up to the virial radius, that would allow to test directly our model (and more generally the scheme invented by CMT). The new generation of satellites (Chandra and XMM-Newton) is expected to bring new observations near the virial radius, allowing to test different formation scenarios of clusters and groups (see e.g., Tozzi et al. 2000).

Nevertheless, some data on the central adiabat value of the gas in groups and clusters are available. Ponman et al. (1999) were the first to show that groups have a central adiabat higher than the one they would have if it had been imposed by shocks only. Recently,

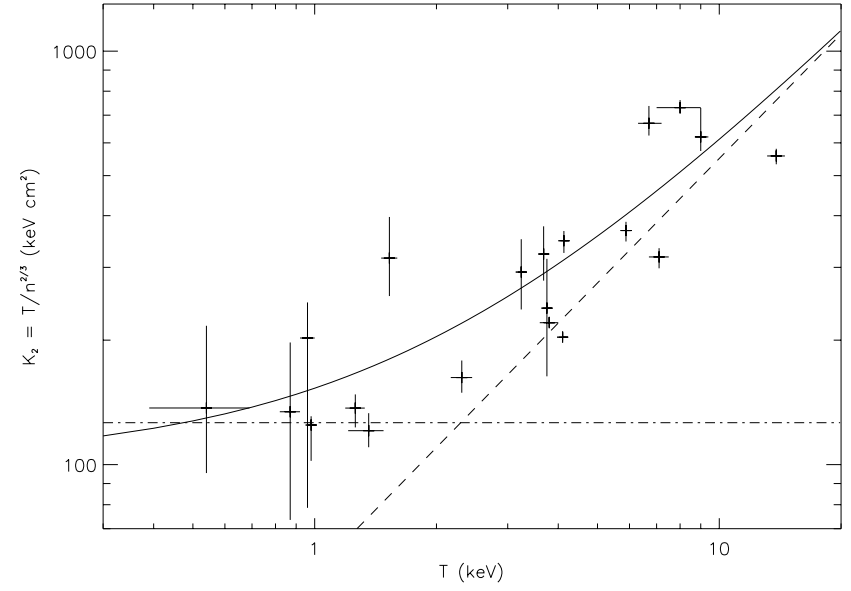

Fig. 2. Post-shock adiabat $K_{2}$ as a function of the mean temperature of the system. The solid line is obtained with Eq. (21), with $K_{1}=120 \mathrm{keV} \mathrm{cm}^{2}$ (i.e., $K_{0}=102 \mathrm{keV} \mathrm{cm}^{2}$ ). Note that only the normalisation is a free parameter, as the reference temperature $T_{0}=2 \mathrm{keV}$ is obtained from the value of $K_{1}$. The dashed line is a $K \propto T$ fit to the systems above $4 \mathrm{keV}$ found by Helsdon \& Ponman (2000). The similarity of the shock model adiabat normalisation (computed at the virial radius) with the data taken at the center $\left(R=0.1 r_{\mathrm{v}}\right)$ allows us to postulate an approximate self-similarity in the specific entropy profile in the Sect. 3.

Lloyd-Davies et al. (2000) have refined these observations, taking into account the temperature gradients, while increasing the statistics. This is the so-called observed "entropy floor". While cool systems have a constant central adiabat, hotter clusters follow naturally the results of numerical simulations without a preheating phase (which predict that $K \propto T$ ). Note that our model predicts exactly the same behaviour, exhibited in the post-shock adiabat analytic expression of Eq. (21). However, we compute the adiabat near the virial radius, while the data come from the central parts of the systems. Nevertheless, observations and theory can be compared if we assume that the adiabat difference between two different temperature systems is conserved when going from the virial radius to the central part. This implies that the physical mechanism responsible for the post-shock adiabat value (competition between shocks and an entropy floor) is the same as the one responsible for the central adiabat value. This is indeed a reasonable assumption, since 3D hydrodynamical simulations of the formation of clusters show that, after the last major merger, a quasi-spherical shock forms and expands, that leaves behind an increasing adiabat profile (see e.g., Evrard 1990; Frenk et al. 1999). The same physics is thus at work in the center and in the outer parts of clusters, allowing the physical description of the two regions to coincide. Indeed, the only difference between these two regions is that the universe will have evolved when the shock reaches the present-day virial radius, as compared to the time when the central entropy is set. We thus don't expect the observational value of $K_{1}$ (found by normalisation to the observations) to be representative of the present 
adiabat of the universe, but it should instead be representative of the IGM at the time when the smaller mass systems formed. If virial radius observations of the adiabat were available for the same systems, we expect then that the same analytic expression as in Eq. (21) would be a good description of the data, with a different value of $K_{1}$, representative of the present-day adiabat of the IGM.

Figure 2 shows the comparison of Eq. (21) with data from Lloyd-Davies et al. (2000). Only the normalisation was fitted here, with a value of $K_{1}=120 \mathrm{keV} \mathrm{cm}^{2}$ (giving $K_{0}=102 \mathrm{keV} \mathrm{cm}^{2}$ ), while we have taken $T_{0}=2 \mathrm{keV}$, derived with the same value of $K_{1}$ in the last section. Replacing Eq. (28) in Eq. (21) instead, and fitting for $K_{1}$ gives an undistinguishible analytic curve. The dashed line is the result of a $K \propto T$ fit to the systems above $4 \mathrm{keV}$ found by Helsdon \& Ponman (2000). This temperature dependence of the adiabat is taken from numerical simulations (see Ponman et al. 1999). The dot-dashed line is a constant gas entropy fitted to the four lowest temperature systems by Lloyd-Davies et al. (2000) and has a value of $139 \mathrm{keV} \mathrm{cm}^{2}$. The agreement between the analytic formula and the data is remarkable. Indeed, using the fitted value of $K_{1}$, the computed $T_{0}$ value agrees very well with the temperature of the intersection between these two limiting behaviours (see last section). This shows that Eq. (21) is not a mere analytic formula (which could have been inferred from the observations), but succeeds in capturing both ingredients which fix the central entropy value: the entropy floor (dot-dashed line in the groups mass range) and the shocks (dashed line, in the cluster mass range).

By adjusting the normalisation of our analytic model using the data we derive a value for $K_{1}$. Then making use of this relation we get directly for any system at a given temperature $T$ (either a group or a cluster) the normalisation of the adiabat profile. It is now important to check whether we are able to use this relation to derive realistic $L_{\mathrm{X}}-T$ and $y-T$ relations for both groups and clusters.

\section{From the adiabat to the $L_{\mathrm{X}} T$ relation}

The total X-ray luminosity of a local cluster (group) of galaxies is expressed as:

$L_{\mathrm{X}}=\int_{\mathrm{V}} n_{\mathrm{e}}^{2} \Lambda_{\mathrm{e}}\left(\Delta E, T_{\mathrm{e}}\right) \mathrm{d} V$

where $n_{\mathrm{e}}$ is the electronic density and the integration is carried out over the whole volume $V$ of the system. $\Lambda_{\mathrm{e}}\left(\Delta E, T_{\mathrm{e}}\right)$ denotes the local X-ray emissivity of the cluster gas within a given energy band $\Delta E$. For example, in the case of pure thermal Brehmsstrahlung emission observed with contemporary wide-band satellite, one has $\Lambda_{\mathrm{e}} \propto T_{\mathrm{e}}^{1 / 2}$.

\subsection{An analytic prediction for the $L_{X}-T$ relation}

We can now express Eq. (29) as a function of $K$ and thus link the X-ray luminosity with the temperature and the adiabat:

$L_{\mathrm{X}}=\int_{\mathrm{V}} \frac{T^{3}}{K^{3}} \Lambda_{\mathrm{e}}(\Delta E, T) \mathrm{d} V$.

Since we assume the cluster to be isothermal and since in general the emissivity can be written as a power-law function of the temperature with index $\alpha$, one has:

$\frac{L_{\mathrm{X}}}{T^{3+\alpha}}=\Lambda_{0} \int_{V} \frac{\mathrm{dV}}{K^{3}}$

$\Lambda_{0}$ being the normalisation of the emissivity.

Equation (31) gives us a direct link between $L_{\mathrm{X}}, T$ and $K$. Were the expression of $K$ as a function of $T$ known, it would enable us to predict the shape of the $L_{\mathrm{X}}-T$ relation. However, the result of the previous section (21) gives us the change in the adiabat normalisation as a function of temperature, but since the integral in Eq. (31) is computed over all the system volume, in principle we need the adiabat profile to compute it. However, if we make the further assumption that the adiabat profiles are self-similar in temperature, i.e. the temperature enters the analytic expression of $K(\boldsymbol{r})$ by its normalisation only, then we can derive an expected $L_{\mathrm{X}}-T$ relation. This assumption is justified by the fact that our theoretical expression for the adiabat normalisation (Eq. (21)), which is computed at the virial radius, provides a very good fit to the central entropy data in groups and clusters. This simple fact ensures that the difference in normalisation between two clusters of different temperature (which is due to shocks and preheating) is leaved approximately unchanged from the virial radius to the center. This important side aspect of our work will be discussed in Sect. 7. We thus write:

$K\left(\boldsymbol{r}, T_{2}\right)=K_{2}\left(T_{2}\right) f(\boldsymbol{r})$

where $K_{2}\left(T_{2}\right)$ is given by Eq. (21) and $f(\boldsymbol{r})$ is a function of the radius, that is independent of the mean system temperature. We have thus:

$\frac{L_{\mathrm{X}}}{T^{3+\alpha}}=\Lambda_{0} K_{2}(T)^{-3} \int_{\mathrm{V}} \frac{\mathrm{d} V}{f^{3}(\boldsymbol{r})}$.

Even if $f$ does not depend on $T$, the integral on the righthand side of the last equation depends on the total volume of the cluster, and thus on its virial radius, which depends also on the mean temperature. Let us assume that the cluster is spherically symmetric (which is reasonable if the system has sufficient time to relax). We can then write:

$$
\begin{aligned}
\int_{V} \frac{\mathrm{d} V}{f^{3}(\mathbf{r})} & =4 \pi \int_{0}^{r_{\mathrm{v}}} r^{2} f^{-3}(r) \mathrm{d} r \\
& =4 \pi r_{\mathrm{v}}^{3} \int_{0}^{1} x^{2} f^{-3}(x) \mathrm{d} x \\
& \equiv 4 \pi r_{\mathrm{v}}^{3} Q_{\mathrm{X}}
\end{aligned}
$$

where we set $x=r / r_{\mathrm{v}}$ and define the shape factor $Q_{\mathrm{X}}$. Using then the $r_{\mathrm{v}}-M$ and $M-T$ relation of Eq. (B.10) 


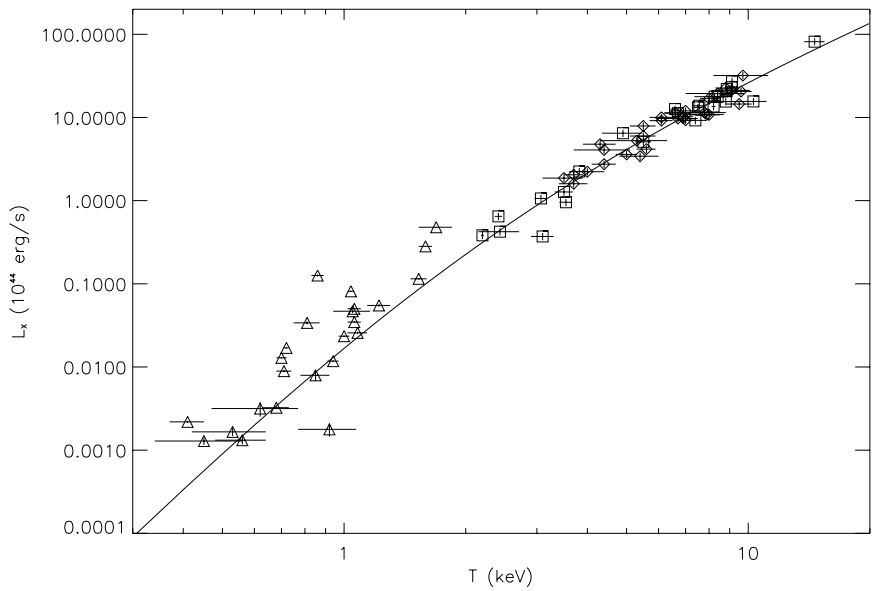

Fig. 3. $L_{\mathrm{X}}-T$ relation versus observations from Helsdon \& Ponman (2000) for groups (triangles) and Markevitch (1998) and Arnaud \& Evrard (1999) for clusters (respectively diamonds and squares). The solid line is the analytic formula in Eq. (37). $T_{0}$ was fixed at the value computed in Sect. 2.3.2 i.e., $T_{0}=2 \mathrm{keV}$, and $Q \mathrm{x}=0.115$, as computed in Appendix C.

and (B.11) before using our $K-T$ relation (21), we obtain when replacing in Eq. (33):

$L_{\mathrm{X}}=L_{0} T^{9 / 2+\alpha}\left[1+\left(\frac{T}{T_{0}}\right)\right]^{-3}$

where the normalisation $L_{0}$ is given by:

$L_{0}=\Lambda_{0}\left(\frac{3 M_{*}}{\Delta \rho_{\mathrm{c}}^{0} T_{*}^{3 / 2}}\right) K_{0}^{-3} Q_{\mathrm{X}}$.

Let us first comment on the shape of the $L_{\mathrm{X}}-T$ relation. While for the group range, where $\alpha=0$ and $T \ll T_{0}$, we have:

$L_{\mathrm{X}} \propto T^{9 / 2} \propto T^{4.5}$

for very hot clusters (for which $T \gg T_{0}$ and $\alpha=1 / 2$ ), we recover the so-called self-similar behaviour:

$L_{\mathrm{X}} \propto T^{9 / 2+1 / 2-3} \propto T^{2}$.

The $10 \mathrm{keV}$ cluster range will be intermediate between these two behaviours. We now turn to a comparison with observations.

\subsection{Comparing with $L_{X}-T$ observations}

The normalisation of the local $L_{\mathrm{X}}-T$ relation (Eq. (38)) depends on the value of the IGM adiabat $K_{0}$ and the shape factor $Q_{\mathrm{X}}$. The former has been obtained in Sect. 2.3.3 $\left(K_{0}=102 \mathrm{keV} \mathrm{cm}^{2}\right)$ by comparing our analytic formula for the adiabat jump to observations. The latter is more difficult to obtain since it will depend on the exact adiabat profile of clusters and groups. We show in Appendix $\mathrm{C}$ that we can compute a value for $Q_{\mathrm{X}}$ by considering a reasonable model for the adiabat profile, relying on isothermality and a $\beta$-model for the gas density profile
(Cavaliere \& Fusco-Femiano 1976). The computed value is $Q_{\mathrm{X}}=0.115$.

To validate this relation, we plot in Fig. 3 the $L_{\mathrm{X}}-T$ relation of Eq. (37) together with data taken for groups (from Helsdon \& Ponman 2000) and clusters of galaxies (Markevitch 1998; Arnaud \& Evrard 1999). The quality of the fit is obviously excellent. Note that we adopt for $T_{0}$ the value computed in Sect. 2.3.2 and used $Q_{\mathrm{X}}=0.115$. There is thus no free parameter in the solid curve computation, since $K_{0}$ has been already fixed.

Note that the hotter groups seem to be overluminous when compared to the cluster data as well as to the analytic relation. There are indeed several uncertainties concerning these systems, both observational and theoretical. Groups are much less luminous than clusters, which has some important consequences: first, the radius of detection (in units of the virial radius) is small and varies among groups: this effect is very hard to correct since the value of the asymptotic slope of the surface brightness $(\beta$ in a $\beta$-model) is very uncertain. Second, it is very difficult to separate the intra-group medium emission from the galactic emission, which can lead to an important misestimation of the total flux (see in particular the discussion on HCG 16 by Dos Santos \& Mamon 1999). Third, the central cooling-flows were cut (Markevitch 1998) or the sample was chosen explicitly to contain only clusters with known weak cooling-flows (Arnaud \& Evrard 1999). On the contrary, the groups luminosity in Helsdon \& Ponman (2000) are not cooling-flow corrected, since it is very delicate to separate the cooling-flow from the ICM emission with ROSAT's PSPC resolution. This could also explain that the hotter groups (which are expected to have the greatest fraction of their luminosity coming from their cooling-flow because of their higher central density) show the greatest departure. Our model does not take into account a possible cooling-flow component, which explains that it fits very well the cluster data, while lying near the lower envelope of the hotter groups. It fits nevertheless very well the lower temperature groups ${ }^{3}$. As usually done, we thus assume that the discrepancies affecting the high temperature groups is mainly due to observational uncertainties and does not compromise our overall simplified picture.

Both the cluster slope and the group steeper slope come out naturally of our relation. This agreement is remarkable and is the first to our knowledge based on an analytic discussion to encompass both groups and clusters.

This good agreements is a strong sign in favor of the consistency of our approach. It is thus natural to try to apply an analogous method to the correlation between the mass of gas and the mean temperature, as well as to the SZ effect, and compare cluster observations with our analytic predictions. Unfortunately, gas mass observations are sparse and heterogeneous for groups, while no SZ effect in a group has yet been observed. We can nevertheless

\footnotetext{
3 For more discussion on this point, see Brighenti \& Mathews (2001), Sect. 2.1.
} 
make predictions for the $M_{\mathrm{ICM}}-T$ and $y-T$ relation in this range, which could be validated by undoubtly forthcoming SZ measurements.

\section{From the adiabat to the ICM gas mass and gas fraction}

\subsection{Predicting the $M_{I C M}-T$ relation and comparing to observations}

Another tight correlation found in reducing ICM data of clusters is the link between the ICM mass and the mean temperature. For a perfectly self-similar model, one expects theoretically $M_{\mathrm{ICM}} \propto T^{3 / 2}$. In fact, several groups have reported recently a steepening of this relation, which could be due to entropy injection. Vikhlinin et al. (1999) have studied the outer regions of a ROSAT sample of clusters, and found that the above correlation could be written $M_{\mathrm{ICM}} \propto T^{1.71 \pm 0.13}$, steeper but nevertheless close to the self-similar value. On the other hand, Mohr et al. (1999) have fitted double $\beta$-models to another large sample of clusters and found that $M_{\mathrm{ICM}} \propto T^{1.98 \pm 0.18}$, much steeper than the self-similar model. It is not clear if this discrepancy (at only $1 \sigma$ level) is real and if so, what are the reasons behind it.

It is easy to compute this correlation in our framework and worth comparing our predictions with observations. The ICM mass of gas can be written (assuming spherical symmetry):

$M_{\mathrm{ICM}}=4 \pi \int_{0}^{r_{\mathrm{v}}} r^{2} \rho_{\text {gas }}(r) \mathrm{d} r$,

where $\rho_{\text {gas }}(r)=\mu m_{\mathrm{p}} n(r)$ is the mass density of plasma. Rescaling with the virial radius and replacing the gas number density by a combination of temperature and adiabat, one finds:

$M_{\mathrm{ICM}}=M_{\mathrm{ICM}}^{0} T^{3}\left[1+\frac{T}{T_{0}}\right]^{-3 / 2}$

with

$M_{\mathrm{ICM}}^{0}=\mu m_{\mathrm{p}}\left(\frac{3 M_{*}}{\Delta \rho_{\mathrm{c}}^{0} T_{*}^{3 / 2}}\right) K_{0}^{-3 / 2} Q_{\mathrm{M}}$

where we defined naturally:

$Q_{\mathrm{M}}=\int_{0}^{1} \frac{x^{2} \mathrm{~d} x}{f^{3 / 2}}$.

The value of this shape factor, which determines the overall normalisation, is also evaluated in Appendix C. The predicted value using Eq. (44) is $Q_{\mathrm{M}}=0.1915$, while the best-fit normalisation is $\sim 40 \%$ lower. We argue in Appendix $\mathrm{C}$ that the observed surface brightness in most of the clusters does not reach $r_{500}$ (the virial radius) and thus needs an risky extrapolation of the data. Moreover, the observational derivation of $r_{\Delta}$ is also dependent on several assumptions (in particular on the assumption of a given mass profile) and it is possible that the gas masses

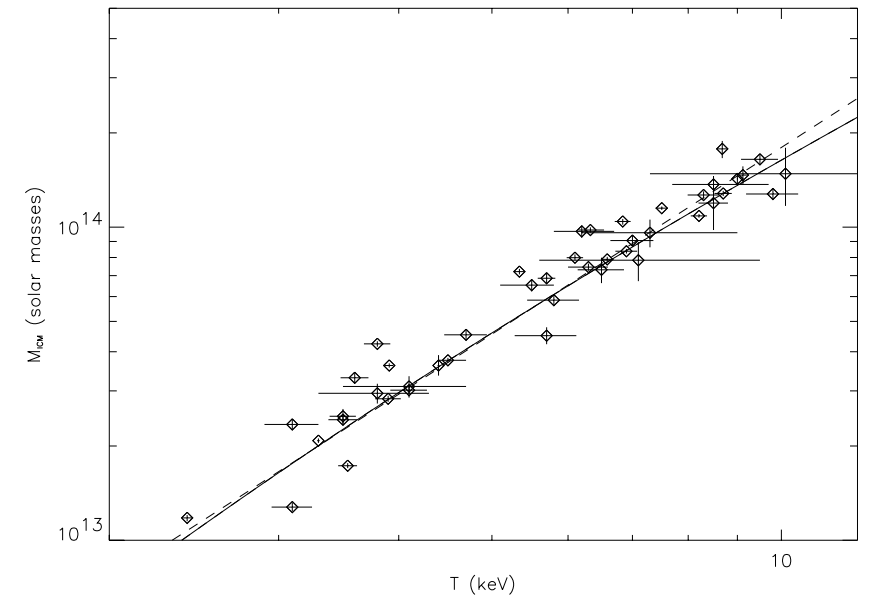

Fig. 4. $M_{\mathrm{ICM}}-T$ correlation. The data are from Mohr et al. (1999). The dashed line is the best-fit linear correlation $M_{\text {ICM }} \propto T^{1.98}$ found by these authors, while the solid line is the predicted correlation (Eq. (42)) using the computed value $Q_{\mathrm{M}}=0.1136$ (see Appendix C). The curvature of our prediction is clearly visible when compared to the best-fit power-law.

were in fact computed at $\Delta \neq 500$. Indeed, lowering the the upper boundary of the integral in Eq. (44) to 0.8 (i.e., assuming $r_{500}$ is underestimated by only $20 \%$ ) gives $Q_{\mathrm{M}}=0.1136$, which is in perfect agreement with the data. We use this value in Fig. 4 and in the following section.

The slope of the correlation also steepens from very hot clusters $\left(M_{\mathrm{ICM}} \propto T^{3 / 2}\right.$, analogous to the self-similar correlation) to groups $\left(M_{\mathrm{ICM}} \propto T^{3}\right)$. The temperature range probed by Mohr et al. (1999) is intermediate between these two behaviours.

Figure 4 shows the data from Mohr et al. (1999), as well as their best-fit linear correlation $M_{\mathrm{ICM}} \propto T^{1.98}$ (dashed line). The solid line is our predicted relation using the computed value $Q_{\mathrm{M}}=0.1136$. The predicted slope matches perfectly the data. The steepening of the relation compared to the self-similar one is thus, in our particular model, simply a consequence of the differential shock strength and the entropy floor.

\subsection{Comparing the subsequent $f_{\text {gas }}-T$ relation with observations}

The gas fraction is very important in that it is supposed to give a direct lower limit on the universe baryon fraction, if clusters are a fair sample of the universe (White et al. 1993). Such measurements have proven to be tight constraints to the total mass density of the universe.

Using Eq. (42) and the observed $M-T$ relation of Eq. (B.11), i.e. the observed scaling between total mass and temperature, it is straightforward to compute the gas fraction variation with temperature:

$f_{\text {gas }}=f_{\text {gas }}^{0} T^{3 / 2}\left[1+\frac{T}{T_{0}}\right]^{-3 / 2}$ 


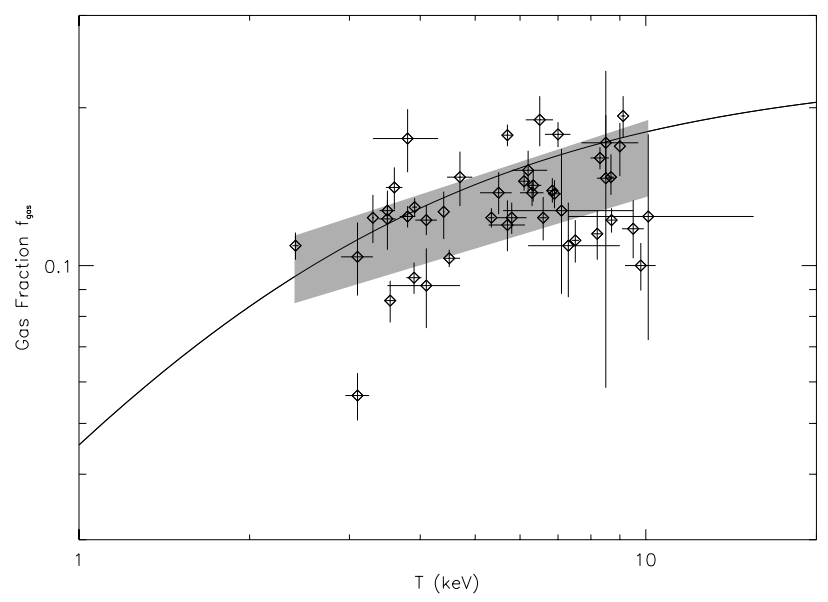

Fig. 5. $f_{\text {gas }}-T$ relation. Data are taken from Mohr et al. (1999) as well as the shaded area which corresponds to the domain of validity of their fitted power law, $\pm 1 \sigma$. The solid line corresponds to our prediction. Both the trend and the normalisation are in good agreements.

with

$f_{\text {gas }}^{0}=\mu m_{\mathrm{p}}\left(\frac{3}{\Delta \rho_{\mathrm{c}}^{0}}\right) K_{0}^{-3 / 2} Q_{\mathrm{M}}$

The value of $Q_{\mathrm{M}}$ is the same as in the last section $\left(Q_{\mathrm{M}}=\right.$ 0.1136 ) and no renormalisation is performed.

Equation (45) is shown in Fig. 5, together with observations. The data points are from Mohr et al. (1999). These data were fitted by the authors with a power-law, namely $f_{\text {gas }}=(0.207 \pm 0.011)(T / 6 \mathrm{keV})^{0.34 \pm 0.22}$. The shaded area, limited in abscissa by the minimum and maximum temperature of the sample, represents the area covered by similar power-laws increased and decreased by $1 \sigma$ (both in normalisation and in slope). It is obvious that the data are dispersed, but the trend found by Mohr et al. (1999) is very well recovered by Eq. (45), as well as the normalisation.

Looking at Eq. (45), one sees that for $T_{0} \ll T$, i.e. for rich clusters, the gas fraction becomes independent of the temperature. This regime is only reached asymptotically in the figure. On the other end the slope steepens from clusters to groups, since we have:

$f_{\text {gas }} \propto f_{\text {gas }}^{0} T_{0}^{3 / 2}=$ constant for rich clusters

$\propto M$ for groups,

where $M$ is the total mass of the group. It is interesting to note that the fact that the gas fraction is proportional to the total mass in the groups regime was first claimed by Balogh et al. (1999). We thus recover analytically the adiabatic behaviour modeled by these authors. However, their model breaks down at high temperature, when the shocks become important, while ours runs smoothly from an adiabatic infall to a shock-dominated regime.

\section{From the adiabat to the SZ temperature decrement}

The observations against which our model was validated above were only in the X-ray wavelengths. We are now turning to the radio and sub-millimeter bandwidth, which also probes the hot gas in clusters (via the SunyaevZeldovich effect) and offers independent observations, with different possible systematics and errors. This allows us to independently validate the model and make some predictions about the group behaviour in this band.

Inverse-Compton scattering of incoming CMB photons on ICM thermal electrons cause a well observed change in the spectral dependence of the CMB (Rephaeli 1995; Birkinshaw 1999). The amplitude of the CMB temperature brightness variation can be written:

$\frac{\Delta T}{T_{\mathrm{CMB}}}=h(x) \int\left(\frac{k T}{m_{\mathrm{e}} c^{2}}\right) n_{\mathrm{e}} \sigma_{T} \mathrm{~d} l \equiv h(x) y$,

where $h(x)$ is the frequency dependence (with $x=$ $\left.h \nu / k T_{\mathrm{CMB}}\right)$, which simplifies to $h(x)=-2$ at the Rayleigh-Jeans part of the spectrum, $y$ being called the comptonization parameter. The integral in the right-hand side of Eq. (48) can be expressed as a function of $K$, as for the X-ray luminosity:

$$
\begin{aligned}
\frac{\Delta T}{T_{\mathrm{CMB}}} & =h(x) y \\
& =h(x) \frac{k \sigma_{T}}{m_{\mathrm{e}} c^{2}} \int n_{\mathrm{e}} T \mathrm{~d} l \\
& =h(x) \frac{\sigma_{T}}{m_{\mathrm{e}} c^{2}} \int \frac{(k T)^{5 / 2}}{K^{3 / 2}} \mathrm{~d} l .
\end{aligned}
$$

\subsection{An analytic prediction for the $y-T$ relation}

For current SZ experiments resolution (especially interferometric ones), the beam smearing is still a critical issue as long as we want to determine a central value. Most authors use X-ray determined temperature as well as the $\mathrm{X}$-ray surface brightness to correct for the beam and to obtain the central value of the SZ effect $y(0)$. This might be a source of uncertainty but we will nevertheless express this quantity in our physical framework, and compare it to observations.

The beam-corrected quantity can be obtained in the following way: going back to Eq. (51) we write (assuming spherical symmetry)

$$
\begin{aligned}
y_{0} & \equiv y(0) \\
& =\frac{\sigma_{T}}{m_{\mathrm{e}} c^{2}} \int \frac{T^{5 / 2}}{K^{3 / 2}(T)} \mathrm{d} l \\
& =\frac{\sigma_{T}}{m_{\mathrm{e}} c^{2}} \frac{T^{5 / 2}}{K_{2}^{3 / 2}(T)} \int_{R}^{\infty} \frac{r \mathrm{~d} r}{\sqrt{r^{2}-R^{2}} f^{3 / 2}(r)} \\
& =\frac{\sigma_{T}}{m_{\mathrm{e}} c^{2}} \frac{T^{5 / 2}}{K_{2}^{3 / 2}(T)} R_{\mathrm{v}} \int_{0}^{\infty} \frac{\mathrm{d} x}{f^{3 / 2}(x)}
\end{aligned}
$$




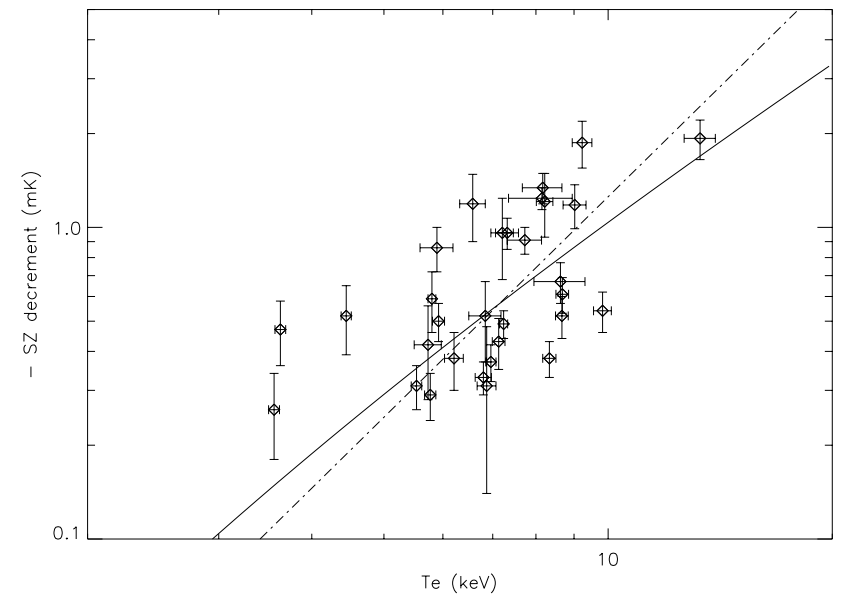

Fig. 6. Central SZ decrement - X-ray temperature relation. The data points are taken from Zhang \& Wu (2000). The solid line is the predicted $y_{0}$ using $Q_{\mathrm{SZ}}=1.835$. The dot-dashed line corresponds to the renormalised correlation fitted by Cooray (1999). The predicted normalisation was computed using $K_{0}=$ $102 \mathrm{keVcm}^{2}$ and $T_{0}=2 \mathrm{keV}$, i.e., it is not free once $K_{0}$ has been fitted to the observations (Sect. 2.3.3).

Using Eqs. (21), (B.10) and (B.11) we have thus:

$y_{0}=\bar{y}_{0} T^{3}\left[1+\left(\frac{T}{T_{0}}\right)\right]^{-3 / 2}$

with

$\bar{y}_{0}=\frac{\sigma_{T}}{m_{\mathrm{e}} c^{2}}\left[\frac{3 M_{*}}{4 \pi \Delta \rho_{\mathrm{c}}^{0} T_{*}^{3 / 2}}\right]^{1 / 3} K_{0}^{-3 / 2} Q_{\mathrm{SZ}}$,

where we have defined:

$Q_{\mathrm{SZ}}=\int_{0}^{\infty} \frac{d x}{f^{3 / 2}(x)}$.

The value of $Q_{\mathrm{SZ}}$ is computed in Appendix C.

Finally, let us make a comment about the shape of the $y-T$ relation. For $T \gg T_{0}$, we get:

$y_{0} \propto T^{3-3 / 2} \propto T^{3 / 2}$,

while for $T \ll T_{0}$ :

$y_{0} \propto T^{3}$.

Thus, as a matter of fact we expect a steepening of the $y-T$ relation when going from clusters to groups.

\subsection{Comparing with $y-T$ observations}

Taking observational data from Zhang \& Wu (2000) (diamonds), we can compare our predicted relation of Eq. (54) to observed correlations.

In Fig. 6 we plot data and their associated error bars, our prediction for $Q_{\mathrm{SZ}}=1.835$ (solid line, computed in Appendix C) and the best fit of (Cooray 1999 dot-dashed line $)^{4}$. The fact that only rich cluster data are yet available (which limits the leverage on the slope) and the large dispersion on the observed $y-T$ correlation translates into a large uncertainty on the best-fit slope: $2.35 \pm 0.85$.

Our prediction for the beam-corrected value is steeper than the Cooray's best-fit relation but still consistent with it at the $1 \sigma$ level. It seems to be in better agreement with Zhang's data set, which extends to slightly higher and lower temperatures than Cooray's. Note that we will further show that Eq. (54) is also in very good agreement with semi-analytic models for both groups and clusters.

The overall agreement with both data sets is less impressive than for the previously derived $L_{\mathrm{X}}-T$ relation, but is still very reasonable, given the observational dispersion.

Since X-ray and SZ observations are completely independent, the agreement between our analytic model with the latter is another independent confirmation of its validity. A robust confirmation would come from SZ observations of groups of galaxies (unfortunately not yet feasible) and from the change in the correlation slopes. Nevertheless, we can compare our simple model to more elaborate semi-analytic models and their predictions.

Menci \& Cavaliere (2001) have presented semi-analytic predictions for the observed SZ effect in groups and clusters. Their semi-analytic model takes into account the preheating due to star formation, in order to bend the $L_{\mathrm{X}}-T$ relation in the groups mass range. Our predictions for the $y(0)-T$ relation (namely Eq. (57) for clusters and (58) for groups) are in complete accord with their preheated models. They found a self-similar relation for high mass systems $\left(y \propto T^{3 / 2}\right)$ which is exactly our prediction. The slope in the groups mass range depends on the feedback model they chose, but our prediction $\left(y(0) \propto T^{3}\right)$ is also similar to their computed moderate feedback model (which looks to be a better choice, since their strong feedback model is likely to give only an upper limit on the feedback effect). We are thus able to reproduce their Figs. 3 and 4 with our simple analytic model. Even if this is not a confirmation of the model (since we use an analytic version of their model of shocks and our hypotheses on the preheating are very similar), we find it extremely satisfying to reproduce analytically, and in a physically straightforward and motivated way, the results of a more complicated and nonanalytic model. This gives us more confidence in the fact, that we have captured in this simple physical scheme the essential ingredients of the clusters and groups formation.

\section{Linking $S Z$ and $X$-ray measurements}

To link SZ and X-ray measurements, it is natural to determine a correlation between $y$ and $L_{\mathrm{X}}$. It is expected to be much tighter than the $y-T$ relation (see Cooray 1999), but is more intricate to obtain analytically. In this section, we will give two analytic expressions for this

\footnotetext{
${ }^{4}$ Note that in Cooray (1999)'s paper, the quoted normalisation of the best-fit $y-T$ relation is wrong by a factor of 10 .
} 


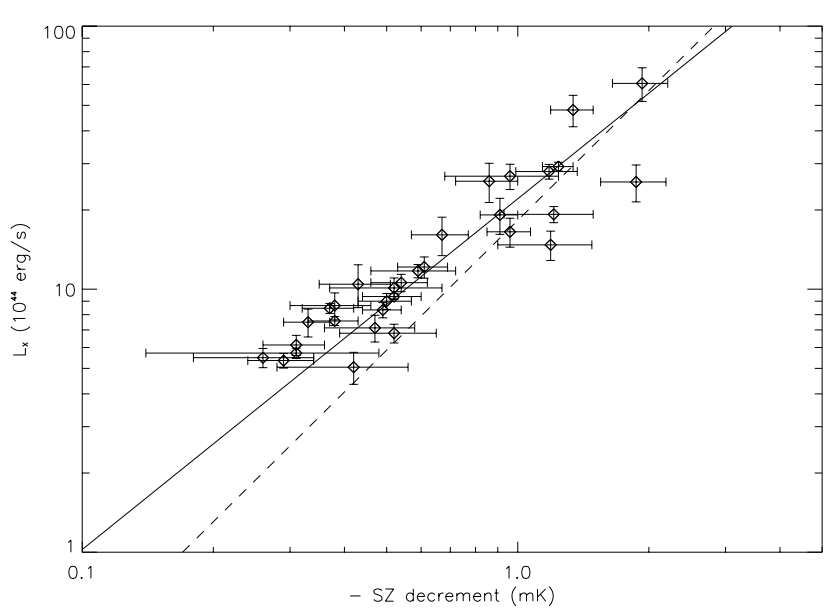

Fig. 7. Central SZ decrement - X-ray luminosity relation. The solid line represents the analytic predicted correlation, using the predicted values of $Q_{\mathrm{Sz}}$ and $Q_{\mathrm{X}}$ found in Appendix C. The dashed line corresponds to Cooray (1999)'s best-fit correlation. The data are taken from Zhang \& Wu (2000).

correlation in the rich clusters's and in the groups' range mass respectively.

Indeed using Eqs. (54) and (37), we can write:

$\frac{L_{\mathrm{X}}}{y_{0}^{2}}=\frac{L_{\mathrm{X}}^{0}}{\bar{y}_{0}} T^{\alpha-3 / 2}$.

To proceed further we need now to "invert" Eq. (54) but this can not be easily done without a new hypothesis. Since the SZ observations span only the range of clusters yet, as long as we want to compare to observations it is satisfying to assume that $T \gg T_{0}$. We can then write:

$T=T_{0}^{-3 / 2}\left(\frac{y_{0}}{\bar{y}_{0}}\right)^{2 / 3}$,

and inserting this in Eq. (59) gives:

$$
\begin{aligned}
L_{\mathrm{X}} & =L_{\mathrm{X}}^{0} T_{0}^{3 / 2}\left(\frac{y_{0}}{\bar{y}_{0}}\right)^{1+\frac{2}{3} \alpha} \\
& =L_{\mathrm{X}}^{0} T_{0}^{3 / 2}\left(\frac{y_{0}}{\bar{y}_{0}}\right)^{4 / 3} \text { with } \alpha=1 / 2 .
\end{aligned}
$$

The observed correlation between $y$ and $L_{\mathrm{X}}$, together with the analytic prediction (Eq. (62), solid line) is shown in Fig. 7. The data are taken from Zhang \& Wu (2000) and the dashed line is the best-fit relation found by Cooray (1999). The normalisation has been computed using $K_{0}=102 \mathrm{keV} \mathrm{cm}{ }^{2}, T_{0}=2 \mathrm{keV}, Q_{\mathrm{X}}=0.115$ and $Q_{\mathrm{SZ}}=1.835$. The agreement is very good, even if it must be recalled that the shown correlation has been computed for the case $T \gg T_{0}$. Thus the real correlation will be slightly steeper (thus more in agreement with Cooray's correlation), but by a very small amount, well within the observational dispersion.

Finally, one can compute this correlation in the groups mass range $\left(T \ll T_{0}\right)$, yielding a steeper correlation:

$L_{\mathrm{X}}=L_{\mathrm{X}}^{0}\left(\frac{y_{0}}{\bar{y}_{0}}\right)^{3 / 2}$ with $\alpha=0$.

\section{Discussion}

We have shown that a simple modeling of the main process in charge of the formation of clusters (namely the shocks which thermalise the gas inside the virial radius) allows us to derive a general $K-T$ relation that reproduces very well the observed one. Moreover, we were able to deduce most of the observed scaling laws with the adequate normalisation, encompassing both groups and clusters. Thus, the physical meaning of e.g. the $L_{\mathrm{X}}-T$ and $y-T$ relations, and in particular of the break in their selfsimilarity when going from clusters to groups (only evidenced in X-rays for the moment) can be understood as a relic of the formation process of these systems: a competition between an entropy floor prior to the shock and the shock process itself. This suggests naturally that entropy constitutes the best "observable" in clusters, as already emphasized by the modified spherical model of Tozzi \& Norman (2001). What we add here is that we show that entropy (in fact, the adiabat) is also suitable to seek analytic expressions for the different correlations found observationally in groups and clusters. Reversing this argument, these scaling laws should be an appropriate probe of the entropy content of clusters.

\subsection{The $L_{X}-T$ relation as a probe of entropy content}

While the entropy spatial variations of the baryonic gas in clusters is a valuable piece of information on their present thermodynamic state (in particular for merging clusters, see e.g., Markevitch et al. 1999), the total entropy content $\bar{K}$ (hereafter TEC), defined as

$\bar{K}=\int_{\mathrm{V}} K(\boldsymbol{r}) \mathrm{d} V$

is related to the integrated thermodynamic history of the formation of these systems. Therefore, any nongravitational entropy injection at some point in this history will enhance the entropy content over the shockgenerated one, while the cooling should decrease it in the central parts.

The analytic model we have presented in the last sections allows us to shed some light in the TEC and its link to the $L_{\mathrm{X}}-T$ relation. On the one hand, given our assumption of isothermality, Eq. (31) indicates that $L_{\mathrm{X}} / T^{3+\alpha}$ is related to the TEC in a given system. On the other hand, the self-similar models of cluster formation (Kaiser 1986) predict that $L_{\mathrm{X}} \propto T^{2}$, which gives directly (using $\alpha=1 / 2)$ :

$\frac{L_{\mathrm{X}}}{T^{7 / 2}} \propto T^{-3 / 2}$.

Any departure from this last relation witnesses entropy injection or loss. Consequently, at a given temperature, the gap between the curve representing Eq. (65) and the locus of observed groups and clusters will give a indirect measure of entropy gain at a given mass (if the central cooling parts are adequately excised). Unlike the observations of 
the "entropy floor", which were obtained at a single scaled radius near the center (Ponman et al. 1999; Lloyd-Davies et al. 2000) and so are representative of a particular point in the entropic history (i.e., at a time near the last major merger), this measure includes most of the volume of the systems and thus most of its past thermodynamic history. However, this does not directly measure $\bar{K}$, but the integral of a power-law of the TEC. We will first show that in a semi-analytic scheme (with less assumptions than the present analytic model as well as with a realistic entropy evolution of the intergalactic medium) the interpretation of the $L_{\mathrm{X}}-T$ relation is still valid and then discuss how $\mathrm{X}$-ray and SZ observations can constraint the TEC.

\subsection{Relaxing some hypotheses}

Assuming local thermodynamic equilibrium, isothermality, and the self-similarity of the adiabat profile, we illustrated how the observed $L_{\mathrm{X}}-T$ relation is a probe of the TEC. Whereas the LTE is quite robust (and actually very difficult in practice to alleviate), neither the isothermality nor the self-similarity are fully exact from both an observational and a theoretical point of view. In particular, this last assumption is a very strong one and will be discussed in depth in Sect. 7.3. It is thus worth relaxing these hypotheses and testing the validity of our work by considering how well the quantity $\int_{\mathrm{V}} K^{-3} \mathrm{~d} V$ probes $L_{\mathrm{X}} / T^{7 / 2}$, i.e. testing Eq. (31) when we consider a full non-isothermal and non-self-similar profile.

To this purpose, we will use a semi-analytic model (hereafter SAM) developed independently by one of us (Dos Santos 2001, in preparation). This particular model uses the conduction-structured temperature profile (which was shown by Dos Santos (2001) to describe very well the temperature and surface brightness profile of clusters of galaxies), together with an NFW profile for the dark matter density profile. A shock model at the virial radius, together with entropic constraints at the center allow to predict the temperature profile without the hypothesis of isothermality or the unphysical polytropic link between temperature and density. The evolution of the central entropy is governed by the entropy evolution of the IGM in the universe obtained from the model of Valageas \& Silk (1999) in two different cases: in the first one, the reheating is provided by SN explosions only, while in the second one, AGN and quasar activity provide the entropy injection. Both cases were validated against a number of observations, including the $L_{\mathrm{X}}-T$ relation, the change in surface brightness profiles from clusters to groups, the baryon fraction in these systems and the entropy floor.

Figure 8 shows the comparison between observations and the SAM. The solid (SN case) and dashed lines (QSO case) show the quantity $\int_{\mathrm{V}} K^{-3} \mathrm{~d} V$ computed directly using the model. Since the specific entropy is known up to an additive constant, both lines were normalised to match the high temperature clusters. The dot-dashed line shows the self-similar prediction for $L_{\mathrm{X}} / T^{7 / 2}$ (Eq. (65)), also

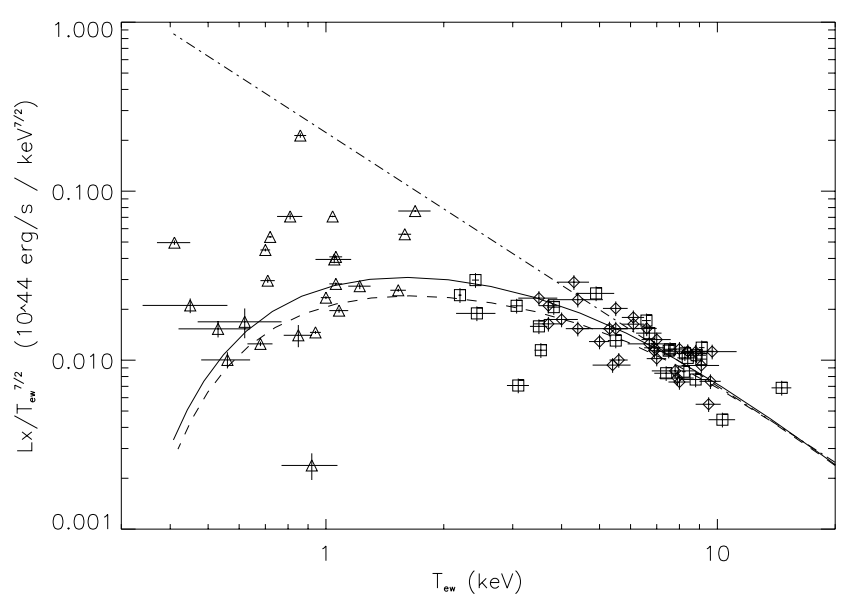

Fig. 8. $L_{x} / T^{7 / 2}$ (observational points) compared to the direct computation of $\int_{\mathrm{V}} K^{-3}(\boldsymbol{r}) \mathrm{d} V$ in the SAM. The data are the same as in the Fig. 3. The solid (respectively dashed) line is the result of the SAM in the case of SN (respectively QSO) reheating. The dot-dashed line is the self-similar prediction $\left(L_{\mathrm{X}} / T^{7 / 2} \propto T^{-3 / 2}\right)$. Thus, relaxing the assumptions of isothermality and self-similarity of our analytic model does not change the interpretation of $L_{\mathrm{X}} / T^{7 / 2}$ as a probe of $\int_{\mathrm{V}} K^{-3}(\boldsymbol{r}) \mathrm{d} V$. This explains that our simple model is able to recover the slope and the normalisation of the $L_{\mathrm{X}}-T$ relation.

renormalised to match rich clusters. The preheated models high temperature slopes match naturally the self-similar prediction. They also match very well the cluster data and their trend in temperature. It is in particular remarkable that the three clusters with the smallest temperatures $(T \sim 2 \mathrm{keV})$ depart notably from the self-similar prediction, and lie exactly on top of the semi-analytic prediction. The lower envelope of groups is well followed by the SAM, while some points are over this prediction. We think that, as in Sect. 3.2, this is due partly to the fact that the central cooling regions of groups were not removed, unlike the clusters, and partly to an intrinsic scatter.

The figure shows why our simple model works well in recovering slope and normalisation of the $L_{\mathrm{X}}-T$ relation (and of the other relations we studied): the equality (31) is also verified in a more general semi-analytic model where the adiabat profile is fully known and the ICM is not isothermal. At a given temperature, the difference in location between observations and the self-similar curve will give a measure of the non-gravitational entropy injection in systems with this mean temperature. This measure does not depend at all on hydrostatic equilibrium or spherical symmetry. Nor does it require either that the preheating was external. In fact, Eq. (33) is completely independent of any modeling of the entropy injection. It only requires isothermality and local thermodynamic equilibrium on the scales probed by the observations. It thus can be used as a powerful, model-independent, constraint on all the internal or external preheating models, as well as on differential galaxy formation efficiency between groups and clusters (Bryan 2000). Unfortunately, the TEC is not measured directly, but the integral of the right-hand side of Eq. (31) will obviously decrease as the entropy injection amount is 
increased. Thus, if the adiabat profile is known in a given model, it will be easy to relate this integral to the TEC, even if a certain amount of degeneracy will obviously be present (this measure being integrated in space and time, different entropy injection histories can lead to the same final amount of entropy at $z=0$ ).

In fact, this constraint will be more or less the same as the constraint given by the $L_{\mathrm{X}}-T$ relation (already used to constrain the models), even if the interpretation in terms of entropy is physically more satisfying. However, the same remarks can be made with the surface integrated SZ decrement. Indeed, the SZ y-Compton parameter integrated over the whole surface of a cluster can be written:

$\frac{y}{T^{5 / 2}}=\frac{\sigma_{T}}{m_{\mathrm{e}} c^{2}} \int_{\mathrm{V}} \frac{\mathrm{d} V}{K^{3 / 2}(\boldsymbol{r})}$,

which shows that the quantity $y / T^{5 / 2}$ is also a probe of the entropy content, but with a different power of the adiabat profile inside the integral. Thus, once SZ observations for groups are available, even non-resolved, the combination of X-rays and SZ at a given temperature will be a powerful constraint on cluster formation and preheating models. Note that the relation $M_{\mathrm{ICM}}-T$ gives an analogous relation as Eq. (66), and can thus be used now as an entropy probe.

\subsection{The adiabat profiles and the self-similarity}

To derive the analytic scaling relations presented in this paper, we have used a strong assumption: the selfsimilarity of rescaled adiabat profiles (see Eq. (32)) i.e., the fact that the temperature enters only the normalisation of the adiabat profile. The validity of this assumption is questionable, both on observational and theoretical grounds.

Ponman et al. (1999) show that density profiles are shallower in groups than in clusters (even if this result is still uncertain, see Roussel et al. 2000, for an alternative view), while preheating models predict naturally that entropy gradients in lower temperature systems are smaller than in clusters (due to adiabatic infall during their lifetime, see Balogh et al. 1999; Tozzi \& Norman 2001). However, these models predict also large temperature gradients in groups which are not observed (Tozzi \& Norman 2001). Moreover, using 1D hydrodynamic models with internal or external preheating, Brighenti \& Mathews (2001) obtain linearly rising entropy profiles in groups (outside an isentropic core for external preheating models) as well as in clusters. They thus produce naturally self-similar adiabat profiles in groups and clusters. The reasons for such discrepancies are unclear, but are certainly linked with widely differing simulation methods. Note however that Knight \& Ponman (1997), using similar 1D hydrodynamic simulations, have found shallower slopes in groups compared to clusters, without taking into account any preheating (whose effect is expected to widen the slope difference).
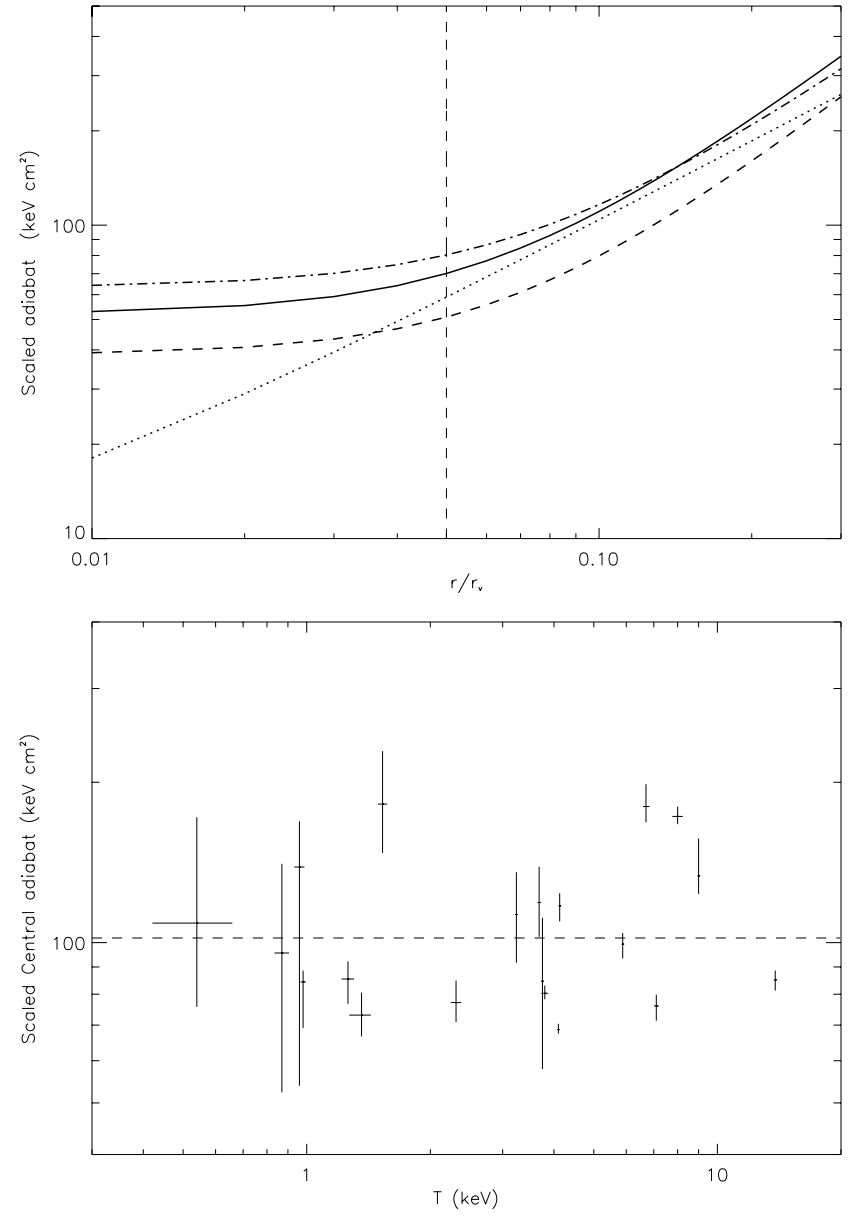

Fig. 9. Upper panel: average scaled adiabat profiles for systems grouped by mean temperature (solid: $6-14 \mathrm{keV}$; dashed: 3.7-6 keV; dot-dashed: 1.3-3.7 keV; dotted: 0.5-1.3 keV). Each individual profile was obtained from the best-fit gas and density profiles given by Lloyd-Davies et al. (2000) and scaled by $\left[1+T / T_{0}\right] /(1+z)^{2}$. No trend is found with the temperature and all the profiles are approximately self-similar. The vertical dashed line shows the mean cooling-flow radius. Bottom panel: scaled central entropy value as a function of the mean temperature of each system. Again, no excess in groups is found, which validates our assumption of self-similar adiabat profiles (at least in the core) and our approach. The dashed line shows the entropy floor value inferred from the data $\left(K_{0}=102 \mathrm{keV} \mathrm{cm}^{2}\right.$, corresponding to $K_{1}=120 \mathrm{keV} \mathrm{cm}^{2}$ ).

Spatially-resolved spectroscopic observations of groups and clusters provide directly adiabat profiles in groups and clusters and can then help to settle this debate. Unfortunately, the low surface-brightness of groups allows this type of study only to small radii ${ }^{5}$. Indeed, LloydDavies et al. (2000) obtained the adiabat profile for 12 systems with $T<4 \mathrm{keV}$ up to $r=0.25 \times r_{\mathrm{v}}$. Even if this maximum extent radius is small, the study of the adiabat profiles behaviour with temperature inside it is still worthwhile, since the X-ray luminosity comes mainly from

\footnotetext{
5 Note that this situation will be much improved with groups observations with XMM-Newton, due to its enhanced sensitivity.
} 
this central part of the cluster (due to the $n^{2}$ scaling of $L_{\mathrm{X}}$ ). These authors have rescaled their observed entropy profiles by a factor $T^{-1}(1+z)^{2}$, removing the effect of system mass and of the evolution of the mean density of the universe. They found that the scaled adiabat profiles normalisations do not coincide, the less massive systems having higher scaled entropy profiles (see their Fig. 4), although the profiles themselves are similar. However, this particular scaling does not take into account the physical processes that change the adiabat normalisation with mass. What we have shown in this paper is that the adiabat profiles of groups and clusters should be rescaled (i.e., divided) by the quantity:

$\left[1+\frac{T}{T_{0}}\right](1+z)^{-2}$,

where the $(1+z)^{-1}$ factor comes from the redshift dependence of the central density (proportional to the mean density of the universe i.e., $\left.\propto(1+z)^{3}\right)$.

Using the best-fit $\beta$-model for the gas density and the best-fit linear ramp for the temperature profile, we reproduced their adiabat profiles, and rescaled them individually by the quantity in Eq. (67). Then we grouped the systems by temperature and computed the mean scaled profile, as in Lloyd-Davies et al. (2000). The scaled profiles are displayed in the upper panel of Fig. 7.3 (we used the same line styles as Lloyd-Davies et al. 2000). The profiles now lie on top of each other, i.e., their normalisation does not depend on the system temperature (as opposed to the result of the scaling in Lloyd-Davies et al. 2000). To confirm this, one can do the same scaling with the central adiabat values (at $r=0.1 \times r_{\mathrm{v}}$ ), which is shown in the bottom panel of Fig. 7.3. Instead of finding an excess for $T<4 \mathrm{keV}$ as in the Fig. 5 of Lloyd-Davies et al. (2000), we find now that all the systems lie on the same central adiabat symbolised by the dashed line and giving directly the value of $K_{0}$ in our model (i.e., $K_{0}=102 \mathrm{keV} \mathrm{cm}^{2}$ ). The slight dispersion can be attributed to the assumption that the observation time equals the formation time of a system. This clearly means that our whole approach of taking into account not only hydrodynamic shocks but also an entropy floor established before them is valid and describes appropriately the dynamics of formation of clusters and groups.

But, the main point of this rescaling is that, without changing the adiabat profiles shape, it allows us to compare them more directly. From the upper panel of Fig. 7.3, it is obvious that the four profiles are very similar between $r=0.05 \times r_{\mathrm{v}}$ (the mean cooling-flow radius, inside which entropy loss is achieved by cooling) and the outer radius. This means that our assumption of scaled self-similar adiabat profiles is indeed right, at least in the core of the systems. This explains why our slope and normalisation work so well for the $L_{\mathrm{X}}-T$ and $y_{0}-T$ relations, which put more weight on the core of the systems. It also enlights the fact that our shape factor for the gas mass is less accurate, since most of the mass lies at large radius where the self-similarity assumption breaks down (see Appendix C).

\subsection{Comparison with other results}

First of all, let us remind the reader that, unlike most of the current models of cluster and group equilibrium, we do not predict the density and temperature profiles. Instead, we are interested in integrated quantities as the luminosity. As such, our model has not the predictive power of some others, but allows us to find analytic expressions for these integrated quantities. In particular, since we assume isothermality and self-similarity of the adiabat profiles, the density profiles do not change with mean temperature (e.g., no evolution of $\beta$ with T), as Ponman et al. (1999)'s analysis would suggest. The satisfying results we obtain show first that the key point is the appropriate modeling of the competition between shocks and an pre-existing entropy floor. Second, this means as well that the prediction of integrated quantities is not very sensitive to the precise knowledge of the profiles and/or that our assumptions are not far from reality (it is known $\beta$ evolves very slightly with temperature).

We have compared most of our analytic results with predictions from semi-analytic models and found good agreement (in particular for the trends of the gas fraction with total mass and the SZ effect with temperature in low temperature systems). Let us now compare the value of the entropy floor we need to reproduce the curvature of the $L_{\mathrm{X}}-T$ relation with values assumed in other theoretical models of energy injection. Balogh et al. (1999) assume a constant entropy injection value of $\sim 350 \mathrm{keV} \mathrm{cm}^{2}$ and Tozzi \& Norman (2001)'s model needs a value in the range 190-960 $\mathrm{keV} \mathrm{cm}^{2}$ in order to steepen $L_{\mathrm{X}}-T$. These values, as noted by Lloyd-Davies et al. (2000), are higher than the observed value, which are likely to be upper limits. On the other hand, our model needs a value of $K_{0}=102 \mathrm{keV} \mathrm{cm}^{2}$, well within the observational range of $70-140 h_{50}^{-1} \mathrm{keV} \mathrm{cm}^{2}$.

As we have indeed fitted this value to the observations, it may be asked if we do not force in fact this agreement. The answer is clearly no, as, first, the agreement of the $K-T$ relation with the observations does not guarantee that the same value of $K_{0}$ will provide the a good description of the other relations (both their shape, via the temperature at which the self-similarity is broken, $T_{0}$, and their normalisation) which range from radio to $\mathrm{X}$ ray data. Second, the other models reproduce as well the $K-T$ relation, together with the $L_{\mathrm{X}}-T$ one (at least Tozzi \& Norman 2001's model is compared to these observations) with the same high value of the entropy floor. A lower value compatible with observations would not fit this relation.

The main difference between our present work and these models is the fact that their low temperature systems are entirely isentropic i.e., no shocks occur at all in groups. This is the claimed approximation made by Balogh et al. (1999), who want to investigate a limiting model (which naturally fails also in the clusters' mass range). However, Tozzi \& Norman (2001) model shocks 
and adiabatic infall, and it is not clear why shocks do not raise the adiabat profiles of their groups.

This clearly shows that shocks can not be completely suppressed in groups unless an unacceptably high entropy floor is needed in order to break the self-similarity in the $L_{\mathrm{X}}-T$ relation. Our model takes shocks and the entropy floor into account by construction both in clusters and groups and is thus able to reproduce nicely all the X-ray correlations with the observed value of $K_{0}$.

\section{Conclusion}

Throughout this paper, we have shown that the adiabat constituted a "key observable" in the ICM, not only because it is a record of the thermodynamic past history of diffuse baryons in groups and clusters, but also because it allowed us to derive for the first time unifying analytic expression for the slope and normalisation of observed correlations of groups and clusters in X-rays and SZ. We have thus derived analytic expressions and their normalisation for the $L_{\mathrm{X}}-T, M_{\mathrm{ICM}}-T, f_{\mathrm{gas}}-T, y_{0}-T$ and $y_{0}-L_{\mathrm{X}}$ correlations (for the latter, two limiting expressions have been provided in the case of groups and of clusters respectively). The Appendix A summarises these correlations and gives the corresponding numerical values of their normalisations. We will conclude by discussing some applications of the model.

Clusters of galaxies, and in particular their baryonic content observed through its X-ray emission, have often been used to provide cosmological constraints (Perrenod 1980; Kaiser 1986, Oukbir et al. 1997; Blanchard et al. 2000 , and references therein). These studies have shown that the cluster population can provide tight constraints, but have also revealed two problems (apart from purely statistical problems due to large error bars of e.g., the temperature in high-redshift clusters): first, the difficulty to model the luminosity in a cluster, mostly due to the fact that it depends on the core properties, whose formation is still uncertain. Second, the small available number of rich clusters, in particular at high redshift, which precludes yet a reliable use of these tests ${ }^{6}$.

We think that the present work, together with the new generation of X-ray observatories, can alleviate both of these problems. First, our model of the cluster luminosity does rely on the physical processes being at work during the formation of a cluster, and not on an hypothetical density profile. Therefore, the luminosity does not depend on an arbitrary core radius and is thus more reliable than previous attempts (in particular, we don't have to assume anything on the core radius evolution like e.g., Romer et al. 2001). Second, we extend the analytic relations to the groups mass scale, taking into account the competition between an external entropy floor and hydrodynamic shocks (thus recovering the curvature of the $L_{\mathrm{X}}-T$

\footnotetext{
${ }^{6}$ It is paradoxical but amusing to note that this same small number of high redshift clusters becomes a powerful test of the total density of the universe (Donahue et al. 1998).
}

relation) and make predictions for other properties of groups. This is useful since the group population is thought (in a hierarchical structure formation cosmology) to be much larger than rich clusters. However, its redshift evolution has never been used to constrain cosmology, partly because of the lack of reliable data and partly because no analytic model of the groups baryonic properties was available (extrapolating the cluster baryonic properties was obviously wrong, as the recent observations show). The first problem will be solved, at least locally, by Chandra and especially XMM-Newton. Our model provides an attempt to solve the second problem. Of course, groups will certainly be much more dispersed than clusters (because the entropy injection will have more effect on them, and any spatial variation of this injection level will affect the properties of a given group), but the number of groups detected will be large: Romer et al. (2001) have estimated that more than 100 clusters with $T>2 \mathrm{keV}$ will be detected in a serendipitous survey for $z<0.2$ (irrespective of the cosmology), a number 10 times higher than for cluster with $T>4 \mathrm{keV}$ and 50 times highers than for $T>6 \mathrm{keV}$ for a survey surface of $\sim 800 \mathrm{deg}^{2}$. The number of groups with $T<2 \mathrm{keV}$ will obviously be superior, certainly not by a factor 10 because of the reduced size and steepening of the $L_{\mathrm{X}}-T$ relation, but at least by a factor of a few. This number of well observed groups (together with pointed observations) will allow an unprecedent local calibration of the group ensemble properties. Using our model, it will be possible to use groups and clusters to constrain cosmology and the cluster LF, TF and $L_{\mathrm{X}}-T$ evolution to add further constraints. Moreover, groups will provide useful constraints on the total amount of reheating the present universe underwent and its redshift evolution if high redshift groups are available (see Sect. 7).

Acknowledgements. We are deeply grateful to Romain Teyssier whose critical reading improved significantly the quality of an earlier draft of this paper. S. Dos Santos aknowledges also very enlightening discussions with G. C. Stewart. The quality and clarity of this paper was also greatly enhanced thanks to the comments of A. Evrard, G. Mamon, T. Ponman and P. Tozzi. Useful comments from F. R. Bouchet and Y. Mellier are also gratefully aknowleged.

\section{Appendix A: Numerical values for the correlations}

In the following appendix, we provide numerical expressions for the normalisations of the different correlations the present model predicts. These will thus be straightforward to use in another context. The temperature $T$ is in $\mathrm{keV}$, and the normalisations were computed using a local Hubble constant of $H_{0}=100 h_{2 / 3} \mathrm{~km} \mathrm{~s}^{-1} \mathrm{Mpc}^{-1}$ with $h_{2 / 3}=2 / 3$. We included the dependence on $h_{2 / 3}$ in each of the following expressions ${ }^{7}$.

We first describe one relation (external to the model and independent from it), the $M-T$ relation, that we

\footnotetext{
${ }^{7}$ We thus have $h_{50}=4 / 3$ and $h_{100}=2 / 3$ where $h_{50}=\left(H_{0} / 50\right)$ and $h_{100}=\left(H_{0} / 100\right)$.
} 
used to relate the virial radius to the temperature. Then, we write the different correlations presented in this work, namely $L_{\mathrm{X}}-T, M_{\mathrm{ICM}}-T, f_{\text {gas }}-T, y_{0}-T$ and $y_{0}-L_{\mathrm{X}}$.

\section{A.1. The mass-temperature relation}

This relation is crucial, in that it allows us to relate the virial radius (where the shock is assumed to take place) with the temperature of the system, via the definition of $M$ in terms of $r_{\mathrm{v}}$. The simulation results of (Evrard et al. 1996) are frequently used for mass-temperature scaling, but they seem to provide systematically a higher normalisation than the observed one, whatever the method used to measure $M$ (Nevalainen et al. 2000 and references therein). We used the observational results of Nevalainen et al. (2000), derived from observed density and temperature profile, because their sample, although small, goes all the way from groups to clusters. These authors give the $M-T$ relation at different scaled radii, from $r_{2000}$ to $r_{500}$ (where $r_{\Delta}$ is the radius whose mean interior density is $\Delta$ times the critical density). Since Evrard et al. (1996) have shown that, inside a radius $r_{500}$, the baryonic gas is in hydrostatic equilibrium to a very good approximation and that hydrostatic masses measured within this radius should be reliable, we choose this radius to normalise the $M-T$ relation. Moreover, while at smaller radius Nevalainen et al. (2000) find that the $M-T$ relation is significantly steeper $\left(99.99 \%\right.$ confidence at $\left.r_{1000}\right)$ than the self-similar prediction $\left(M \propto T^{3 / 2}\right)$, the slope they measure at $r_{500}$ is consistent with $3 / 2\left(\chi^{2} / \nu=5.1 / 5\right.$, but note that the best fit has a slope of 1.84$)^{8}$. We thus used throughout the paper the following relation:

$$
\begin{aligned}
M_{500} & =M_{*}\left(\frac{T}{T_{*}}\right)^{3 / 2} \\
& =9.6 \times 10^{14} M_{\odot} h_{2 / 3}^{-1}\left(\frac{T}{10 \mathrm{keV}}\right)^{3 / 2},
\end{aligned}
$$

which defines the values $M_{*}$ and $T_{*}$ used in the text. Each time we speak about the virial radius in this paper, we refer to the radius $r_{500}$ related to $M_{500}$ defined above, and we will thus take $\Delta=500$ throughout.

Finally, let us state that introducing a steeper slope for the $M-T$ relation is straightforward within the physical scheme we used in this work, but does not change much the predicted relations gathered in this appendix and their agreement with observations. Since we wanted to discuss the generic effect of shocks and preheating, the same effects which are thought to steepen the $M-T$ relation, we found natural to keep a self-similar scaling.

\section{A.2. The predicted $X-S Z-T$ correlations}

We now compute the different normalisations given in Eqs. (38), (43), (46), (55) and (62). For this purpose, we

\footnotetext{
8 Note that the slope at $r_{500}$ was obtained using only rich clusters, since groups are too faint to allow a detection this far from the centre.
}

will use the values of $M_{*}, T_{*}$ and $\Delta$ from the last section. The critical density is defined as usual as:

$\rho_{\mathrm{c}}^{0}=\frac{3 H_{0}^{2}}{8 \pi G}$,

and, for the luminosity, we will need the normalisation of the cooling function, taken from Eke et al. (1998):

$$
\begin{aligned}
\Lambda_{\mathrm{e}}(T) & =\Lambda_{0} T^{1 / 2} \\
& =1.2 \times 10^{-24} T^{1 / 2} \mathrm{erg} \mathrm{cm}^{3} \mathrm{~s}^{-1},
\end{aligned}
$$

where $T$ is in $\mathrm{keV}$.

Finally, we take for $T_{0}$ the value computed from $K_{0}$ in the Sect. 2.3.2 i.e., $T_{0}=2 \mathrm{keV}$ and the values of the shape factors $Q_{\mathrm{X}}, Q_{\mathrm{SZ}}$ and $Q_{\mathrm{M}}$ computed in Appendix C. Therefore, the only adjusted quantity used to produce these relations is $K_{0}$, which was fitted to the data of Helsdon \& Ponman (2000) in Sect. 2.3.3 to find $K_{0}=102 \mathrm{keV} \mathrm{cm}^{2}$. Once this quantity was fixed by comparison with the central entropy observations, no renormalisation of the following relations is allowed.

All the numbers are computed here at $z=0$, but it is easy, within a given cosmology, to extend the formulae in the text to higher redshift (note that the value of $K_{0}(z)$ must be assumed as well). The analytic correlations found are then

$$
\begin{aligned}
& \text { - } \boldsymbol{L}_{\mathbf{X}}-\boldsymbol{T} \text { relation: } \\
& L_{\mathrm{X}}=5.643 \times 10^{42} T^{5}\left[1+\frac{T}{T_{0}}\right]^{-3} h_{2 / 3}^{-2} \mathrm{erg} \mathrm{s}^{-1}
\end{aligned}
$$

- $M_{\mathrm{ICM}}-T$ relation:

$$
\begin{gathered}
M_{\mathrm{ICM}}=2.405 \times 10^{12} T^{3}\left[1+\frac{T}{T_{0}}\right]^{-3 / 2} h_{2 / 3}^{-2} M_{\odot} \\
-\boldsymbol{f}_{\text {gas }}-\boldsymbol{T} \text { relation: } \\
f_{\text {gas }}=0.0792 T^{3 / 2}\left[1+\frac{T}{T_{0}}\right]^{-3 / 2} h_{2 / 3}^{-1}
\end{gathered}
$$

- $\mathrm{y}_{0}-\mathrm{T}$ relation:

$y_{0}=3.506 \times 10^{-6} T^{3}\left[1+\frac{T}{T_{0}}\right]^{-3 / 2} h_{2 / 3}^{-2 / 3}$,

which can be translated in central temperature decrement as (using $T_{\mathrm{CMB}}=2.73 \mathrm{~K}$ and the RJ approximation)

$$
\begin{aligned}
\Delta T(0)= & -1.914 \times 10^{-2} T^{3} \\
& \times\left[1+\frac{T}{T_{0}}\right]^{-3 / 2} h_{2 / 3}^{-2 / 3} \mathrm{mK} .
\end{aligned}
$$

- $\Delta T(0)-L_{\mathrm{X}}$ relation:

$\star$ for $\boldsymbol{T} \gg \boldsymbol{T}_{\mathbf{0}}$ (rich clusters temperature range):

$$
\begin{aligned}
L_{\mathrm{X}}= & 2.206 \times 10^{45}\left(\frac{-\Delta T(0)}{1 \mathrm{mK}}\right)^{4 / 3} \\
& \times h_{2 / 3}^{-10 / 9} \mathrm{erg} \mathrm{s}^{-1}
\end{aligned}
$$




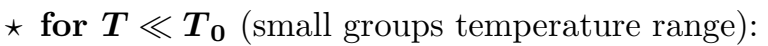

$$
\begin{aligned}
L_{\mathrm{X}}= & 2.133 \times 10^{45}\left(\frac{-\Delta T(0)}{1 \mathrm{mK}}\right)^{3 / 2} \\
& \times h_{2 / 3}^{-1} \mathrm{erg} \mathrm{s}^{-1}
\end{aligned}
$$

\section{Appendix B: Infall velocity and mean temperature}

In this Appendix, we will find an expression for the infall velocity before the shock (noted $v_{1}$ ) as a function of the mean temperature of the accreting system. To this purpose, the following reasonning in two steps is appropriate: we first show that $v_{1}^{2} \propto \phi_{\mathrm{v}}$ provides a good approximation and then derive a usefull expression for $\phi_{\mathrm{v}}$.

We will then compare our result to hydrodynamic simulations, which will validate the use of the analytic expression found in the groups mass range.

\section{B.1. Reexpressing the infalling velocity}

Let's assume that the gas inflow is stationary. In a spherical collapse model, the gas is assumed to be at rest at a radius $r_{\mathrm{ta}}$ (the so-called "turn-around radius") before falling into the cluster potential. Since the gas will not be subjected to many processes changing its internal energy during this inflow, we can assume that the flow is isentropic. Thus, applying the Bernouilli equation between $r_{\mathrm{ta}}$ and $r_{\mathrm{v}}$ (just before the shock) we obtain (see e.g. Landau \& Lifshitz 1959):

$\frac{v_{1}^{2}}{2}+\omega_{1}+\phi_{\mathrm{v}}=\omega_{\mathrm{ta}}+\phi_{\mathrm{ta}}$

where $\omega$ denotes the gas specific enthalpy $(\omega=$ $c_{\mathrm{p}} T$ with $c_{\mathrm{p}}$ the specific heat at constant pressure). Denoting $\rho_{\text {ta }}$ and $\rho_{1}$ the densities at $r_{\text {ta }}$ and $r_{\mathrm{v}}$ and, the following expression for $v_{1}$ can easily be found:

$v_{1}^{2}=-2 \phi_{\mathrm{v}}\left[1-\frac{\phi_{\mathrm{ta}}}{\phi_{\mathrm{v}}}+c_{p} \frac{T_{1}}{\phi_{\mathrm{v}}}\left(1-\left(\frac{\rho_{\mathrm{ta}}}{\rho_{1}}\right)^{2 / 3}\right)\right]$.

In the spherical collapse model, the density at $r_{\mathrm{v}}$ is $\sim 8$ times larger than at $r_{\mathrm{ta}}$.

We can thus write:

$v_{1}^{2}=-2 \phi_{\mathrm{v}}\left[1-\frac{\phi_{\mathrm{ta}}}{\phi_{\mathrm{v}}}+\frac{c_{\mathrm{p}}}{4} \frac{T_{1}}{\phi_{\mathrm{v}}}\right]$.

Obviously, in large mass systems, the last ratio will be negligible i.e., the thermal energy of the gas will be negligible when compared to its potential energy (the so-called "cold inflow" hypothesis). As gas is preheated (i.e., as $T_{1}$ rises) or as the mass of the system is lowered this assumption is questioned, since the thermal content of the gas will be of the same order of the kinetic and potential energy. Nevertheless, we will assume in the following that this limiting behaviour only occurs at mass scales smaller than groups of galaxies. We will show at the end of the appendix that hydrodynamic numerical simulations of structure formation in different cosmological models validate this last assumption.
We have thus:

$v_{1}^{2}=-2 \phi_{\mathrm{v}}\left(1-\frac{\phi_{\mathrm{ta}}}{\phi_{\mathrm{v}}}\right)$.

To take into account some uncertainties and shortcomings of this crude treatment (as the fact that the infall is usually along filaments and so not spherical), we will write the slightly more general formula:

$v_{1}^{2}=-2 \eta \phi_{\mathrm{v}}$,

where $\eta$ is considered as a constant. $\eta$ could be calibrated against numerical simulations (see e.g., Miniati et al. 2000) or chosen so that the flow at infinite is the Hubble flow, as in Cavaliere et al. (1998). When used to compute the value of $T_{0}$ in Sect. 2.3.2, $\eta$ is determined by the former method.

Note that the established fact that the square of the infall velocity is proportionnal to the gravitational potential, both taken at the virial radius, is actually not a surprise. Indeed, before the shock, the gas is thought to follow the dark matter evolution, its velocity being equal to the dark matter one up to the virial radius (see for example the Fig. 15 of Frenk et al. 1999). Since it has been shown that the dark matter infall velocity in a spherical model scales as $M^{1 / 3}$, while the mean potential $\phi \propto M / R$ scales as $M^{2 / 3}$ (Ryu \& Kang 1997, based on the work of Bertschinger 1985), it is thus natural that $v_{1}^{2} \propto M^{2 / 3} \propto \phi$.

\section{B.2. Linking $\phi_{v}$ with $T$}

We now look for a useful expression for $\phi_{\mathrm{v}}$ without assuming any particular analytic expression for $\phi(\boldsymbol{r})$. First, since the Poisson equation states:

$\nabla^{2} \phi=4 \pi G \rho$,

we can write any gravitational potential in the form:

$\phi(x)=4 \pi G \rho_{\mathrm{c}} r_{\mathrm{v}}^{2} \tilde{\phi}(x)$,

where $\rho_{\mathrm{c}}$ is a characteristic density (the background cosmological density for example), $r_{\mathrm{v}}$ is the virial radius, $G$ the gravitational constant and $\tilde{\phi}(x)$ is a dimensionless potential with $x=r / r_{\mathrm{v}}$. Therefore we have in particular,

$\phi_{\mathrm{v}}=4 \pi G \rho_{\mathrm{c}} r_{\mathrm{v}}^{2} \tilde{\phi}(1)$.

By using this as a general relation, we assumed implicitely that $\tilde{\phi}(1)$ does not depend on the mass of the system. However, numerical simulations indicate that the general expression for the gravitational potential is indeed universal (i.e. it takes the same form in different cosmological models), but its normalisation should depend slightly on the mass via the so-called concentration parameter $c$ (Navarro et al. 1997, hereafter NFW). Indeed, using the so-called NFW analytic expression, $\phi_{\mathrm{v}}$ can be expressed as (see for example Łokas \& Mamon 2001) ${ }^{9}$ :

$\phi_{\mathrm{v}}=4 \pi G \rho_{\mathrm{c}} r_{\mathrm{v}}^{2} g(c)$.

${ }^{9}$ Note that the function $g(c)$ defined here differs from the one adopted by Łokas \& Mamon (2001). 
Since in these models, $c$ is a slowly varying function of the mass $\left(c(M)=\left(M / M_{15}\right)^{-0.086}\right.$ for a $\Lambda$ CDM universe at $z=0, M_{15}$ being the mass in units of $\left.10^{15} M_{\odot}\right)$ it is easily shown that $g(c) \propto T^{0.012}$, that is to say, $g(c)$ can be considered as constant to a very good approximation. As a consequence, ignoring the mass dependency of $\tilde{\phi}(1)$ in Eq. (B.8) is reasonnable and we will thus use Eq. (B.8) as a definition of the virial radius gravitational potential.

An even more practical form for Eq. (B.8) does involve the (supposed isothermal) ICM temperature $T$. It is achievable through the use of the virial theorem. It indeed provides us with general relations between $r_{\mathrm{v}}-M$ and $M-T$ in the following form:

$$
\begin{aligned}
& r_{\mathrm{v}}=\left(\frac{3 M}{4 \pi \Delta \rho_{\mathrm{c}}}\right)^{1 / 3} \\
& M=M_{*}\left(\frac{T}{T_{*}}\right)^{3 / 2}
\end{aligned}
$$

where $\Delta$ is the virial radius overdensity (taken throughout this paper to be 500, see Appendix A), $\rho_{\mathrm{c}}$ is the critical density and $M_{*}$ and $T_{*}$ are constants defined in Appendix A. As a conclusion, we can now write Eq. (B.8) as:

$$
\begin{aligned}
\phi_{\mathrm{v}} & =4 \pi G \rho_{\mathrm{c}} \tilde{\phi}(1)\left(\frac{3 M_{*}}{4 \pi \Delta \rho_{\mathrm{c}}}\right)^{2 / 3}\left(\frac{T}{T_{*}}\right) \\
& =\phi_{1}\left(\frac{T}{T_{*}}\right) .
\end{aligned}
$$

Eventually, by combining Eqs. (B.13) and (B.5) we reach the following relation describing the scaling of $v_{1}^{2}$ with $T$ :

$v_{1}^{2}=-2 \eta \phi_{1}\left(\frac{T}{T_{*}}\right)$.

Equation (B.14) is particularly interesting since it can be directly compared to the the results of hydrodynamic numerical simulations. Miniati et al. (2000) have examined the properties of shock waves around clusters and groups of galaxies in two hydro $+N$-body realisations of two different cosmological models. Having selected all structures with X-ray luminosity greater than $10^{41} \mathrm{erg} / \mathrm{s}$, they found that the relation between the mean temperature and the infall velocity is given by an equation of the form (we only quote their result for the $\Lambda \mathrm{CDM}$ cosmology, analogous expressions being found in the SCDM cosmology):

$v_{\mathrm{s}}=v_{\mathrm{s} 0}\left(\frac{T}{T_{\mathrm{s}}}\right)^{0.52}$.

with

$v_{\mathrm{s} 0}=1.9 \times 10^{3} \mathrm{~km} \mathrm{~s}^{-1}$ and

$T_{\mathrm{s}}=7.8 \times 10^{7} \mathrm{~K}$

Their result spans a range from $10^{6}$ to several $10^{7} \mathrm{~K}$, i.e. from poor groups to clusters, and is analogous to
Eq. (B.14) ${ }^{10}$. This, together with the fact that secondary infall models predict the same type of relation as the one we deduced between $v_{1}$ and $\phi_{\mathrm{v}}$, validates the assumption of cold inflow that was made in deriving Eq. (B.5), even for the group mass range.

\section{Appendix C: The shape factors $Q_{\mathrm{X}}, Q_{\mathrm{Sz}}$ and $Q_{\mathrm{M}}$.}

In this appendix, we evaluate the shape factors $Q_{\mathrm{X}}, Q_{\mathrm{SZ}}$ and $Q_{\mathrm{M}}$, which enter the normalisation of the $L_{\mathrm{X}}-T$, $y-T$ and $M_{\mathrm{ICM}}-T$ relations. These quantities could in fact be considered as renormalisation constants obtained by comparison with observations. We will instead show here that they can be computed using a motivated model for the adiabat profile. The model we present thus predicts not only the shape but also the normalisation of the above correlations.

\section{C.1. Normalisation temperature}

The normalisations of the three relations cited above are defined in Eqs. (38), (42) and (54), where the temperature is in $\mathrm{keV}$. The temperature at which the normalisation must be evaluated is obtained by equating the temperature shape function in each of these relations to 1 . This is illustrated in Fig. C.1, which shows the following functions (the last two ones being the same):

$L_{\mathrm{X}}(T)=\frac{T^{5}}{\left[1+T / T_{0}\right]^{3}}($ solid line $)$

$y(T)=\frac{T^{3}}{\left[1+T / T_{0}\right]^{3 / 2}}$ (dashed line $)$

$M_{\mathrm{ICM}}(T)=\frac{T^{3}}{\left[1+T / T_{0}\right]^{3 / 2}}($ dashed line $)$,

and their intersection with the horizontal unit line. We can see that the normalisation temperature of the three relations occurs at $T \sim 1.3 \mathrm{keV}$. These temperatures are well into the groups temperature range, and we will thus compute the normalisation in this regime. First of all, we need an motivated analytic adiabat profile to compute the integrals defining the three normalisation constants.

\section{C.2. An analytic model for the adiabat profile}

Groups have a low surface brightness in X-rays and are thus difficult to observe up to their virial radius. Obtaining a temperature profile at this radius is obviously even more challenging. While the new generation of X-ray satellites (Chandra and XMM-Newton in particular with its exceptional sensitivity) is expected to produce constraints on low density regions, ROSAT (whose energy band was perfectly adapted to the groups observation) has provided data mostly on the inner parts of these systems (see

\footnotetext{
10 We will take the exponent of Eq. (B.15) to be $1 / 2$.
} 


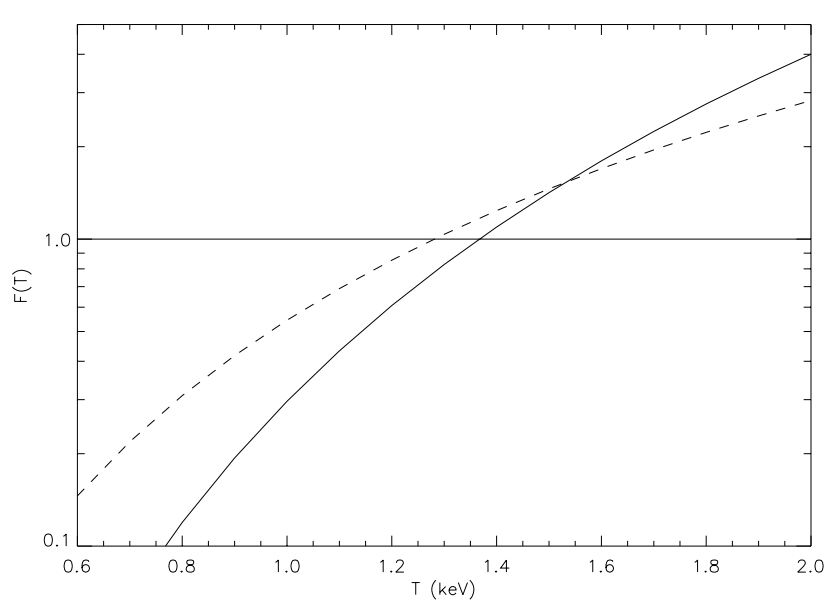

Fig. C.1. Normalisation temperature of the $L_{\mathrm{X}}-T$ (solid line), $y-T$ and $M_{\mathrm{ICM}}-T$ (dashed line) relations. The three relations will thus be normalised at $T \sim 1.3 \mathrm{keV}$, in the groups mass range.

e.g., Helsdon \& Ponman 2000). Nevertheless, David et al. (1996) have published adiabat profiles for two cool clusters $(T \sim 1.5 \mathrm{keV})$ nearly reaching the virial radius (as well as for even cooler groups, see their Fig. 10), using a $\beta$-model for the density and the best-fit power-law model for the temperature (despite large error bars). They found a isentropic core, followed by a very modest increase compared to clusters (where the virial value of the adiabat is at least 20 times higher than the core value).

This can be easily understood, if one recalls that groups are thought to accrete most of their gas adiabatically, and should thus have constant adiabat profiles (Balogh et al. 1999; Tozzi \& Norman 2001). However, this produces unacceptably large temperature gradients in small groups and the quasi-absence of the coolingflow phenomenon, except in the largest clusters (Tozzi \& Norman 2001, see their Figs. 4c and 7b). It shows that somewhere between the center and the virial radius, shocks must increase somewhat the adiabat, in order to lower the temperature gradient, giving rise to an adiabatic inner core (much larger than the core radius) followed by a modestly increasing adiabat profile ${ }^{11}$.

One can seek an analytic expression for the adiabat profile by making use of the isothermal $\beta$-model (Cavaliere \& Fusco-Femiano 1976). Suppose that the gas density profile is describe by this model i.e.,

$n(x)=n_{0}\left[1+\left(\frac{x}{x_{0}}\right)^{2}\right]^{-3 \beta / 2}$,

\footnotetext{
11 Note that HCG62, one of the best examples of relaxed group $(\bar{T} \sim 1 \mathrm{keV})$, has a steep declining temperature outside the inner core (see Fig. 4 of Finoguenov \& Ponman 1999), but has also an obvious cooling-flow.
}

where the radii are rescaled in units of $r_{\mathrm{v}}$ and $x_{0}$ is the core radius. Assuming isothermality, the dimensionless adiabat profile is then

$f(x)=\left[1+\left(\frac{x}{x_{0}}\right)^{2}\right]^{\beta}$.

Using the semi-analytic model already discussed in the text (Dos Santos 2001, in preparation, see Sect. 7.2), we found that, in an external preheated model (by QSOs or $\mathrm{SNs}$ ), the adiabat profile of groups and clusters can be described approximately by the last equation with $\beta=1 / 2$ and a core radius varying with mass. Even if the systems modelled are not isothermal, this result is not surprising as the shocks are expected to leave a linearly rising adiabat profile (hence the value of $\beta$, see Thomas \& Couchman 1992; Tozzi \& Norman 2001; Brighenti \& Mathews 2001), while the isentropic accretion of gas leaves a constant adiabat profile. Fixing $\beta$ to $1 / 2$, the core radius $x_{0}$ determines the extend of the central isentropic core. We have found that $x_{0}=0.7$ reproduces approximately the adiabat profile of small groups in our semi-analytic model, while $x=0.1$ reproduces its behaviour in rich clusters. This is in line with the observational results of David et al. (1996) showing that the profiles are much shallower in groups than in clusters. Thus we will use $\beta=1 / 2$ and $x_{0}=0.7$ in Eq. (C.2) to compute the values of the shape factors.

\section{C.3. The shape factor $Q_{X}$}

The theoretical shape factor is defined by:

$$
\begin{aligned}
Q_{\mathrm{X}} & =\int_{0}^{1} \frac{x^{2} \mathrm{~d} x}{f^{3}(x)} \\
& =\int_{0}^{1} x^{2}\left[1+\left(\frac{x}{0.7}\right)^{2}\right]^{-3 / 2} \\
& \sim 0.1150 .
\end{aligned}
$$

This value is very close to the best-fit value $(0.12$, considering $Q_{\mathrm{X}}$ as an adjustable parameter) and undistinguishable given the observational errors. Although the normalisation has been computed in the groups mass ranges, it recovers perfectly the slope and normalisation of rich clusters. We will thus use $Q_{\mathrm{X}}=0.115$.

\section{C.4. The shape factor $Q_{S Z}$}

The shape factor for the $y-T$ relation is given by:

$$
\begin{aligned}
Q_{\mathrm{SZ}} & =\int_{0}^{\infty}\left[1+\left(\frac{x}{0.7}\right)^{2}\right]^{-3 / 4} \mathrm{~d} x \\
& \sim 1.835 .
\end{aligned}
$$

Here again the normalisation is in very good agreement with the observational data from Zhang \& Wu (2000), despite the large dispersion of the latter (see Fig. 6). 


\section{C.5. The shape factor $Q_{M}$}

The shape factor for the $M_{\mathrm{ICM}}-T$ relation is given by:

$$
\begin{aligned}
Q_{\mathrm{M}} & =\int_{0}^{1} x^{2}\left[1+\left(\frac{x}{0.7}\right)^{2}\right]^{-3 / 4} \\
& \sim 0.1915 .
\end{aligned}
$$

The best-fit value to the data of Mohr et al. (1999 hereafter MME) is $Q_{\mathrm{M}}=0.11$ i.e., $\sim 40 \%$ lower than the value we compute. However, apart from pure measurements uncertainties and known biases ${ }^{12}$, several systematic effects can cause this disagreement: first, the major part of the 45 clusters they study are not detected up to the radius $r_{500}$. Thus, they extrapolate the data taking their best-fit slope out to $r_{500}$, which can be a source of errors. Second, as MME explain, computing $r_{500}$ requires a model of the potential or a rescaling to the value of a given cluster (they chose A1795 for the scaling of their virial relations). If one of the assumptions made is erroneous, it is possible that their numbers quote a radius $r_{\Delta}$ with $\Delta \neq 500$. Indeed, if $r_{500}$ is underestimated by only $20 \%$, we obtain:

$$
\begin{aligned}
Q_{\mathrm{M}} & =\int_{0}^{0.8}\left[1+\left(\frac{x}{0.7}\right)^{2}\right]^{-3 / 4} \\
& \sim 0.1136,
\end{aligned}
$$

which is very close to the best-fit value. We use this last value for $Q_{\mathrm{M}}$ in the $M_{\mathrm{ICM}}-T$ relation, as well as in the $f_{\text {gas }}-T$ relation.

The luminosity of a cluster is dominated by the core gas (since it scales as the square of the density) and the SZ effect computed here is a central value (taking into account the beam smearing effect), while the gas mass depends mainly on the outer parts of the profile, where ourassumption of self-similarity of the scaled adiabat profiles is more likely to break down (see Sect. 7.3). This explains naturally why the predictions for $Q_{\mathrm{X}}$ and $Q_{\mathrm{SZ}}$ are much more accurate than for $Q_{\mathrm{M}}$. Nevertheless, we consider the agreement between the theoretical values and the observed normalisations very satisfying. The fact that with the same model for the adiabat profile we can predict the correct normalisations of the cluster correlations in two very different wavebands is obviously a sign of coherence of the whole scheme. Since the shape factors are predicted, the only parameter of the model is the central entropy in groups $K_{0}$, which was fit to the observations of central adiabat value.

\section{References}

Arnaud, M., \& Evrard, A. E. 1999, MNRAS, 305, 631 Balogh, M. L., Babul, A., \& Patton, D. R. 1999, MNRAS, 307, 463

\footnotetext{
12 Note that testing their analysis method with hydrodynamic numerical simulations, MME find that they are likely to overestimate the ICM mass by a factor of $\sim 12 \%$, which increases the disagreement with our $Q_{\mathrm{M}}$ value by the same amount.
}

Balogh, M. L., Pearce, F. R., Bower, R. G., \& Kay, S. T. 2001, MNRAS, accepted [astro-ph/0104041]

Bertschinger, E. 1985, ApJS, 58, 39

Birkinshaw, M. 1999, Phys. Rep., 310, 97

Blanchard, A., Sadat, R., Bartlett, J. G., \& Le Dour, M. 2000, A\&A, 362, 809

Blanchard, A., Valls-Gabaud, D., \& Mamon, G. A. 1992, A\&A, 264, 365

Bower, R. G. 1997, MNRAS, 288, 355

Bower, R. G., Benson, A. J., Lacey, C. G., et al. 2000, MNRAS, accepted [astro-ph/0006109]

Brighenti, F., \& Mathews, W. G. 2001, ApJ, 553, 103

Bryan, G. L. 2000, ApJ, 544, L1

Cavaliere, A., \& Fusco-Femiano, R. 1976, A\&A, 49, 137

Cavaliere, A., Menci, N., \& Tozzi, P. 1997, ApJ, 484, L21

Cavaliere, A., Menci, N., \& Tozzi, P. 1998, ApJ, 501, 493

Cavaliere, A., Menci, N., \& Tozzi, P. 1999, MNRAS, 308, 599

Cooray, A. R. 1999, MNRAS, 307, 841

David, L. P., Jones, C., \& Forman, W. 1996, ApJ, 473, 692

Donahue, M., Voit, G. M., Gioia, I., et al. 1998, ApJ, 502, 550

Dos Santos, S. 2001, MNRAS, 323, 930

Dos Santos, S., \& Mamon, G. A. 1999, A\&A, 352, 1

Eke, V. R., Navarro, J. F., \& Frenk, C. S. 1998, ApJ, 503, 569

Evrard, A. E. 1990, ApJ, 363, 349

Evrard, A. E., \& Henry, J. P. 1991, ApJ, 383, 95

Evrard, A. E., Metzler, C. A., \& Navarro, J. F. 1996, ApJ, 469, 494

Finoguenov, A., \& Ponman, T. J. 1999, MNRAS, 305, 325

Frenk, C. S., White, S. D. M., Bode, P., et al. 1999, ApJ, 525, 554

Helsdon, S. F., \& Ponman, T. J. 2000, MNRAS, 315, 356

Kaiser, N. 1986, MNRAS, 222, 323

Kaiser, N. 1991, ApJ, 383, 104

Knight, P. A., \& Ponman, T. J. 1997, MNRAS, 289, 955

Landau, L. D., \& Lifshitz, E. M. 1959, Fluid mechanics, Course of theoretical physics (Oxford: Pergamon Press 1959)

Lloyd-Davies, E. J., Ponman, T. J., \& Cannon, D. B. 2000, MNRAS, 315, 689

Loewenstein, M. 2000, ApJ, 532, 17

Łokas, E. L., \& Mamon, G. A. 2001, MNRAS, 321, 155

Markevitch, M. 1998, ApJ, 504, 27

Markevitch, M., Forman, W. R., Sarazin, C. L., \& Vikhlinin, A. 1998, ApJ, 503, 77

Markevitch, M., Sarazin, C. L., \& Vikhlinin, A. 1999, ApJ, 521,526

Miniati, F., Ryu, D., Kang, H., et al. 2000, ApJ, 542, 608

Mohr, J. J., Mathiesen, B., \& Evrard, A. E. 1999, ApJ, 517, 627

Navarro, J. F., Frenk, C. S., \& White, S. D. M. 1997, ApJ, 490, $493+$

Nevalainen, J., Markevitch, M., \& Forman, W. 2000, ApJ, 532, 694

Olive, K. A., Steigman, G., \& Walker, T. P. 2000, Phys. Rep., 333, 389

Oukbir, J., Bartlett, J. G., \& Blanchard, A. 1997, A\&A, 320, 365

Perrenod, S. C. 1980, ApJ, 236, 373

Ponman, T. J., Cannon, D. B., \& Navarro, J. F. 1999, Nature, 397, 135

Rephaeli, Y. 1995, ARA\&A, 33, 541

Romer, A. K., Viana, P. T. P., Liddle, A. R., \& Mann, R. G. 2001, ApJ, 547, 594

Roussel, H., Sadat, R., \& Blanchard, A. 2000, A\&A, 361, 429 
Ryu, D., \& Kang, H. 1997, MNRAS, 284, 416

Sarazin, C. L. 1988, X-ray emission from clusters of galaxies, Cambridge Astrophysics Series (Cambridge: Cambridge University Press)

Teyssier, R. 2001, submitted to A\&A

Teyssier, R., Chièze, R., \& Alimi, J. 1997, ApJ, 480, 36

Thomas, P. A., \& Couchman, H. M. P. 1992, MNRAS, 257, 11

Tozzi, P., \& Norman, C. 2001, ApJ, 546, 63

Tozzi, P., Scharf, C., \& Norman, C. 2000, ApJ, 542, 106
Valageas, P., \& Schaeffer, R. 2000, A\&A, 359, 821

Valageas, P., \& Silk, J. 1999, A\&A, 350, 725

Vikhlinin, A., Forman, W., \& Jones, C. 1999, ApJ, 525, 47

White, S. D. M., Navarro, J. F., Evrard, A. E., \& Frenk, C. S. 1993, Nature, 366, 429

Wu, K. K. S., Fabian, A. C., \& Nulsen, P. E. J. 2000, MNRAS, 318,889

Zhang, T., \& Wu, X. 2000, ApJ, 545, 141 